\title{
Energy Scheduling of Community Microgrid with Battery Cost using Particle Swarm Optimisation
}

\author{
Md Alamgir Hossain ${ }^{\mathrm{a}, \mathrm{b}}$, Hemanshu Roy Pota ${ }^{\mathrm{a}}$, Stefano Squartini ${ }^{\mathrm{c}}$, Forhad Zaman ${ }^{\mathrm{a}}$, Josep M.Guerrero ${ }^{\mathrm{d}}$ \\ ${ }^{a}$ SEIT, University of New South Wales (UNSW), Canberra, Australia \\ ${ }^{b}$ Department of EEE, Dhaka University of Engineering \& Technology (DUET), Gazipur, Bangladesh \\ ${ }^{c}$ Department of Information Engineering, Università Politecnica delle Marche, Ancona, Italy \\ ${ }^{d}$ Department of Energy Technology, Aalborg University, Aalborg, Denmark
}

\begin{abstract}
The integration of renewable energy sources together with an energy storage system into a distribution network has become essential not only to maintain continuous electricity supply but also to minimise electricity costs. The operational costs of this paradigm depend highly upon the optimal use of battery energy. This paper proposes day-ahead scheduling of the battery energy while considering its degradation costs due to chargingdischarging cycles. The degradation costs with respect to the depth of charge are modelled and added to the objective function to determine the actual operational costs of the system. A framework to solve the function is developed in which particle swarm optimisation, the Rainflow algorithm and scenario techniques are integrated. Uncertainties of parameters, modelled by scenario generation and reduced by scenario reduction techniques, are discussed. Simulation results demonstrate that the proposed method can reduce the operational costs by around $40 \%$ compared to the baseline method. They also reveal that uncertainty in power generation and power demand has no influence on the energy schedule of the battery, but variation in electricity prices has an impact on the outcome. Several pragmatic tests verify the effectiveness of the proposed method.

Keywords: Community microgrids, scheduling battery energy, battery degradation costs, renewable energy and optimisation algorithms.
\end{abstract}

\section{Nomenclature}

$\begin{array}{llll}\text { RESs } & \text { Renewable energy sources } & \text { PV } & \text { Photovoltaic } \\ \text { EMS } & \text { Energy management system } & P_{w T} & \text { Total wind power } \\ \text { TOU } & \text { Time-of-use } & P_{s T} & \text { Total solar power } \\ \text { RTP } & \text { Real-time electricity price } & P_{g} & \text { Import/export grid power } \\ \text { ESS } & \text { Energy storage system } & C(t) & \text { Grid electricity prices } \\ \text { ADP } & \text { Approximate dynamic programming } & L(t) & \text { Load at time } t \\ \text { PSO } & \text { Particle swarm optimisation } & B L_{o} & \text { Initial battery energy level } \\ \text { HBB-BC } & \text { Hybrid big bang big crunch } & B L_{\max } & \text { Maximum battery energy level } \\ \text { SCADA } & \text { Supervisory control and data acquisition } & B L_{\min } & \text { Minimum battery energy level } \\ \text { SI } & \text { Solar irradiation } & P_{c, \text { max }} & \text { Maximum charging rate }\end{array}$

Email addresses: Md.Hossain6@student.adfa.edu.au or alamgir_duet@hotmail.com (Md Alamgir Hossain), h.pota@unsw.edu.au (Hemanshu Roy Pota), s.squartini@univpm.it (Stefano Squartini), f.zaman@unsw.edu.au (Forhad Zaman), joz@et.aau.dk (Josep M.Guerrero) 


$\begin{array}{llll}P_{d, \text { max }} & \text { Maximum discharging rate } & \text { EOFP } & \text { Existing objective function with penalty method } \\ u & \text { Battery command signals } & \text { EOFR } & \text { Existing objective function with repairing method } \\ \text { BC } & \text { Battery purchasing cost } & \text { POFP } & \text { Proposed objective function with penalty method } \\ \text { BS } & \text { Battery size } & \text { POFR } & \text { Proposed objective function with repairing method } \\ \text { MC } & \text { Maintenance cost } & \text { ETOF } & \text { Expected time to operational failure } \\ \text { CRF } & \text { Capital recovery factor } & \text { FPA } & \text { Flower pollination algorithm } \\ \eta & \text { Efficiency } & \text { ALO } & \text { Ant lion optimizer } \\ C F & \text { Constriction factor } & \text { GA } & \text { Genetic algorithm } \\ \phi & \text { Constriction co-efficient } & \text { NBAT } & \text { Novel bat algorithm } \\ r & \text { The annual real interest rate } & \text { ADHDP } & \text { Action dependent heuristic dynamic programming } \\ n & \text { Lifetime of a battery } & \text { Nor } & \text { Normal distribution } \\ v & \text { Wind velocity } & \text { STD } & \text { Standard deviation }\end{array}$

\section{Introduction}

Increasing penetration of renewable energy sources (RESs) into an existing distribution network is a driving force to develop a microgrid as an intelligent and modern power system [1. A microgrid (consisting of smallscale emerging generators, loads, energy storage elements and a control unit) is a controlled small-scale power system that can be operated in an islanded and/or grid-connected mode in a defined area to facilitate the provision of additional power and/or maintain a standard service [2]. Intelligent control units or an energy management system (EMS) is responsible for its secure, economical and systematic operation while addressing microgrid challenges, such as uncertainty in power generation and power demand [3]. These uncertainties result in vulnerability in the network operation.

One of the methods for managing network power efficiently is electricity prices that can be set in either a static or dynamic way by the network operator [4]. Static pricing schemes, for example, fixed prices or time-ofuse (TOU) prices, are not affected by the network conditions, such as high/low power generation. Fixed prices do not change the users' pattern, but TOU prices can shift load patterns from high peak to low peak hours by providing the incentive of marginal prices. However, these schemes may not work effectively in future due to the existence of high uncertainty in generating renewable power. For example, if direct sunlight is not available for two or three days, then it will be challenging to balance power in the network without massive power curtailment. With RESs power, it becomes essential to consider dynamic pricing schemes, such as real-time electricity price (RTP) that is often announced hours ahead by the network operators [5. This announcement helps users to schedule flexible loads, such as washing machine use, for minimising electricity costs. Customers can also get benefit from exchanging energy with the grid utility.

Another way to deal with the uncertainty is to integrate an energy storage system (ESS), such as a battery, into a microgrid [6]. It can store or release energy at any time due to flexible charging-discharging characteristics and therefore can be used to provide mismatch energy in the microgrid. ESS can also be used to store and export energy to provide ancillary services and financial benefits to investors, depending upon user demands. Although ESS has numerous beneficial features to employ in a microgrid, one of the key challenges of its application is its high investment cost [7]. Most importantly, it has a limited life span, much shorter than 
RESs, leading to higher replacement costs. Therefore, it becomes crucial while scheduling battery energy to consider the degradation costs of a battery that are related to operational performance to estimate the real operational cost of energy management.

Battery energy is generally managed in two ways: real-time operation and scheduling operation $\underline{3}$. In real-time operation, the battery operates based on the available real-time measurement of resources, whereas scheduling operation presets the battery commands depending on prediction. Generally, this prediction is executed for 24 hours, i.e., day-ahead scheduling [8]. Scheduling battery energy ensures improved power supply security and reliability by proper energy management. This also minimises the operational costs of the microgrid. Thus, for this study, the scheduling program is chosen although it is a difficult task due to variable RESs, loads and electricity prices over the periods.

In the literature, several optimisation algorithms are applied to control battery energy optimally [ㅇ, 9, 10, 11, 12, 13]. In [8], stochastic programming for a day-ahead operation of home energy management systems is presented, and it is shown that the combination of thermal and electrical storage can reduce operating costs. A day-ahead scheduling problem considering TOU for buying/selling electricity is studied using interval optimisation for a grid-connected microgrid in [9]. In [10], an optimisation model for the day-ahead management of a multi-building district is presented. The study shows the impact of considering the degradation costs of a battery for improving battery life and cost-efficient management. A two-stage energy management system, using approximate dynamic programming (ADP) and hybrid big bang big crunch (HBB-BC) algorithms, considering TOU tariffs and controllable loads to manage power generation and reduce operational costs is presented in [1]. In [12, a control strategy with different optimisation schemes for a day-ahead scheduling approach of a PV-battery system is developed and several charging goals formulated as objective functions are solved using dynamic programming. Action dependent heuristic dynamic programming (ADHDP) is developed in [13] for optimal use of battery energy to minimise electricity costs and energy waste. In [14], ADHDP for renewable energy and battery management is employed to manage the energy efficiently by minimizing costs. The study implements a real-time operation of the energy sources without including battery cost. In [15], residential energy scheduling of solar energy considering its different features, such as sunny and cloudy, is presented to increase energy efficiency. Adaptive dynamic programming to efficiently manage electricity consumption of an office building through battery control is presented in [16]. In the study, various schemes for different types of rooms, such as computer and meeting rooms, to minimise electricity consumption are developed, but the degradation costs of a battery are not taken into account. An error-tolerant iterative adaptive dynamic programming algorithm to solve optimal battery control and management problems in smart home environments with renewable energy is developed in [17. The study formulates the problem to keep the battery energy at the middle of the nominal values without justifying true operational costs.

The majority of the literature reported has considered either different parameters or optimisation algorithms for energy management of a microgrid. Although various objective functions with constraints are developed, their explicit impacts on the system, especially degradation costs of a battery and its optimal energy use, are not demonstrated. In addition, the existing traditional optimisation techniques could not solve this complex problem with a real degradation cost function. Therefore, this paper proposes day-ahead energy management of a microgrid in which the battery degradation costs are modelled to consider their effects on the operational costs of the microgrid. To solve the problem formulated, a framework is developed, where a particle swarm 
optimisation (PSO) algorithm is used. The algorithm is compared with other recently developed algorithms to demonstrate its effective performance in solving the energy management problem. The major contributions of this study are given below.

1. The degradation cost model of a battery is developed to determine the real operational cost of the microgrid. As this model accurately calculates the degradation cost by applying the Rainflow algorithm, it helps to reduce electricity bills.

2. A framework to apply for the energy scheduling program of the microgrid is developed in which the PSO algorithm, the Rainflow algorithm and scenario generation techniques are integrated.

3. Mathematical models are presented for solar generators, wind generators, batteries and the grid utility to facilitate computational analysis.

4. The proposed energy management scheme is tested under different scenarios, such as RESs, loads and electricity prices, to prove its effectiveness.

The remainder of the paper is organised as follows. In Section 2, an overview of a community microgrid is introduced. Section 3 presents the models of microgrid components, such as solar and wind generators, to facilitate analysis. In Section 4 a degradation cost model of a battery is proposed to control the battery energy optimally in the microgrid scheduling program. Section 5 develops a framework of solution approaches using algorithms for the energy scheduling problem presented. The experimental simulations of the microgrid considering different scenarios are discussed in Section 6 and Section 7 concludes with the findings of the study.

\section{Microgrid overview}

A community of fifteen houses, located in a remote area with available natural resources, has built a community microgrid as shown in Fig. 1. The microgrid is connected to the main grid through the point of common coupling (PCC) and a transformer. Although it is capable of operating in either islanded or grid-tied mode, only the latter is considered in this study. Both the grid and RESs can supply power to the local loads and charge the battery simultaneously. The energy of the microgrid can be exchanged with the grid utility in order to reduce operational costs. This microgrid is similar to that described in [18, 19]. It is worth mentioning that our previous study in [19] compares various cost functions and proposes a novel cost function to carry out real-time energy management of the microgrid, whereas the paper in [18 proposes two-hour ahead real-time energy management considering battery degradation costs. The work presented in this study is a day-ahead energy scheduling of the microgrid in which problem formulations and analyses are totally different from that of the previous works. A fair comparison of the above energy managements is beyond the scope of this study and left for future work.

The microgrid consists of $40 \mathrm{~kW}$ wind generation, $35 \mathrm{~kW}$ solar PV generation, and a $40 \mathrm{~kW}$ h battery system. The minimum and maximum working capacities of the battery are limited to $20 \%$ and $90 \%$ of its rated capacity, respectively. Solar irradiation and wind velocity profiles of the community are demonstrated in Figures 2 and 3, respectively. The load pattern of an individual house is shown in Figure 4. The total loads of each house can be divided into critical and non-critical loads to avoid total power outage during power supply shortage. The 
peak demand of a house is approximately $2.2 \mathrm{~kW}$ as shown in Figure 4 . In this paper, the size of the storage system is chosen to meet the peak demand for at least one hour to reduce investment costs although this size can be varied depending on the requirements. This small size increases the complexity of energy management due to its limited capacity. To coordinate a microgrid's different components in a systematic way for ensuring its secure, reliable and economical operation, an EMS is used as shown in Figure 1.

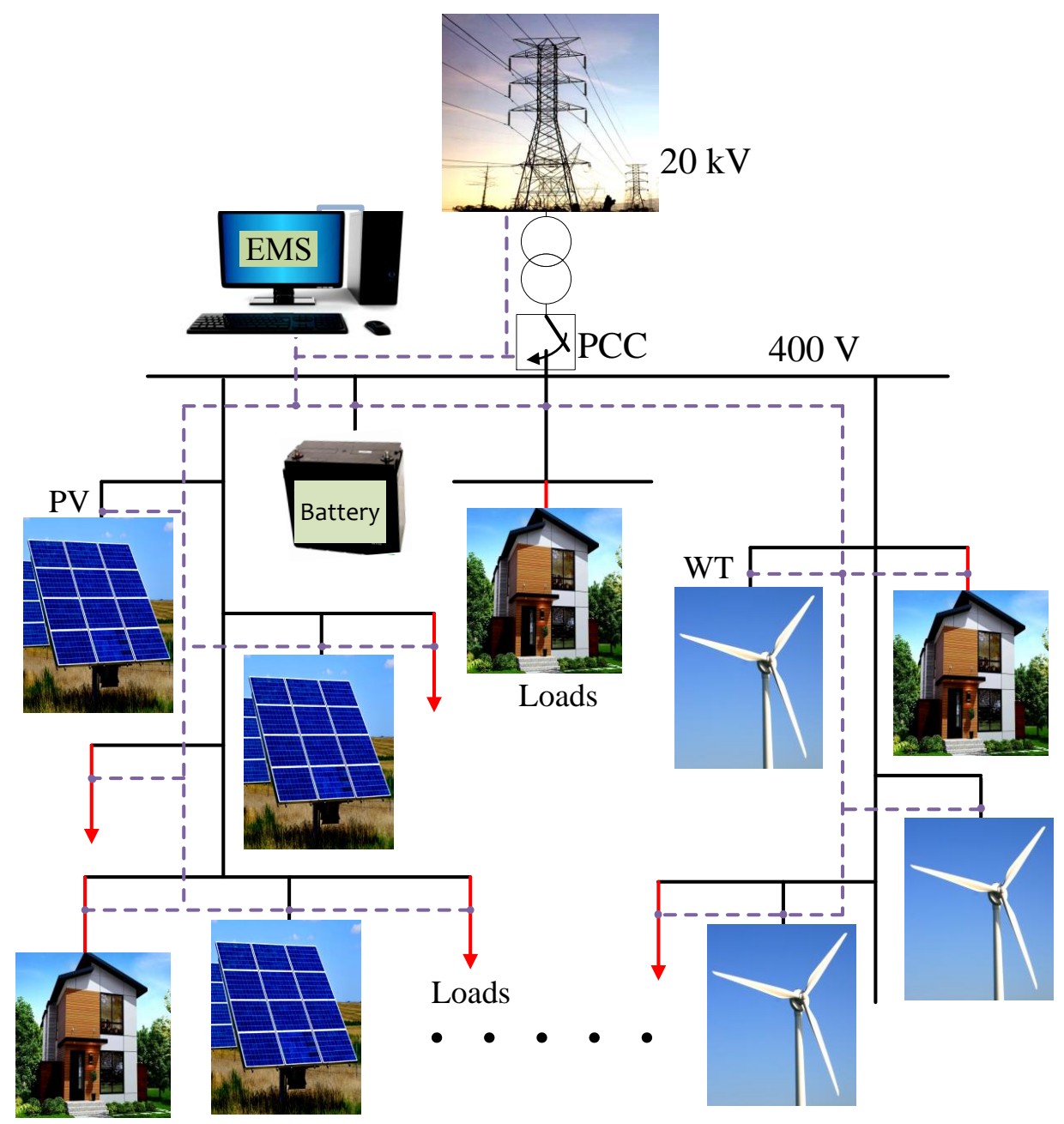

Figure 1: A studied community microgrid.

An EMS is a physical computer system consisting of a software platform in which various modules for generation and load forecasts, human-machine interfaces and supervisory control and data acquisition (SCADA) are used [3]. It generally executes several processes for forecasting power generation, power demand and electricity market prices, and for monitoring, analysing and optimising data to provide the functions necessary for the effective operation of a power system by sending optimal decisions in order to ensure power supply at minimum costs. Based on the forecast of input parameters, such as power generation and demand, day-ahead scheduling is generally carried out for determining power set points of resources to minimise operational costs. In this study, we adopt day-ahead scheduling of the microgrid for the predicted input values. 
Table 1: Input parameters.

\begin{tabular}{|c|c|c|}
\hline Parameter & Value & Unit \\
\hline \multicolumn{3}{|l|}{ PV generators } \\
\hline Covered area, $\mathrm{A}$ & 34 & $\mathrm{~m}^{2}$ \\
\hline Efficiency, $\eta_{s}$ & 16 & $\%$ \\
\hline Maximum power & 5 & $\mathrm{~kW}$ \\
\hline No of PV panels & 7 & \\
\hline \multicolumn{3}{|l|}{ Wind generators } \\
\hline Cut-in velocity & 3 & $\mathrm{~m} / \mathrm{s}$ \\
\hline Cut-out velocity & 25 & $\mathrm{~m} / \mathrm{s}$ \\
\hline Rated speed & 12 & $\mathrm{~m} / \mathrm{s}$ \\
\hline Maximum power & 5 & $\mathrm{~kW}$ \\
\hline No of wind turbines & 8 & \\
\hline \multicolumn{3}{|l|}{ Battery } \\
\hline Energy capacity & 40 & $\mathrm{kWh}$ \\
\hline Maximum energy level, $B L_{\max }$ & 36 & $\mathrm{kWh}$ \\
\hline Minimum energy level, $B L_{\min }$ & 7.2 & $\mathrm{kWh}$ \\
\hline Initial energy level, $B L_{o}$ & 18 & $\mathrm{kWh}$ \\
\hline Maximum charging rate & 4 & $\mathrm{kWh}$ \\
\hline Maximum discharging rate & -4 & $\mathrm{kWh}$ \\
\hline Cost of battery & 680 & $\$ / \mathrm{kW} \mathrm{h}$ \\
\hline Maintenance cost & 20 & $\%$ \\
\hline Interest rate & 6 & $\%$ \\
\hline Lifetime & 10 & years \\
\hline
\end{tabular}




\section{System modeling}

\subsection{Solar generators}

The output power of the PV panels, depending on their size and efficiency, can be determined as a function of solar irradiation (SI) as follows:

$$
P_{s}=\eta_{s} * A * S I\left(1+\gamma\left(t_{o}-25\right)\right)
$$

where $\eta_{s}$ and $A$ are the overall efficiency and area of PV panels, respectively, $t_{o}$ and $\gamma$ denote outside air temperature and the temperature coefficient of the maximum output power, respectively. The value of $\gamma$, depending on the PV technology and manufacturing parameters, is considered as $-0.005 /{ }^{0} C$ for this study. The range of $\gamma$ for silicon cells is $0.004-0.006$ per ${ }^{0} C$ [20].

For a number of solar generators, the total output power can be extracted as follows:

$$
P_{s T}=P_{s} \times N_{s}
$$

where $N_{s}$ is the number of solar generators.

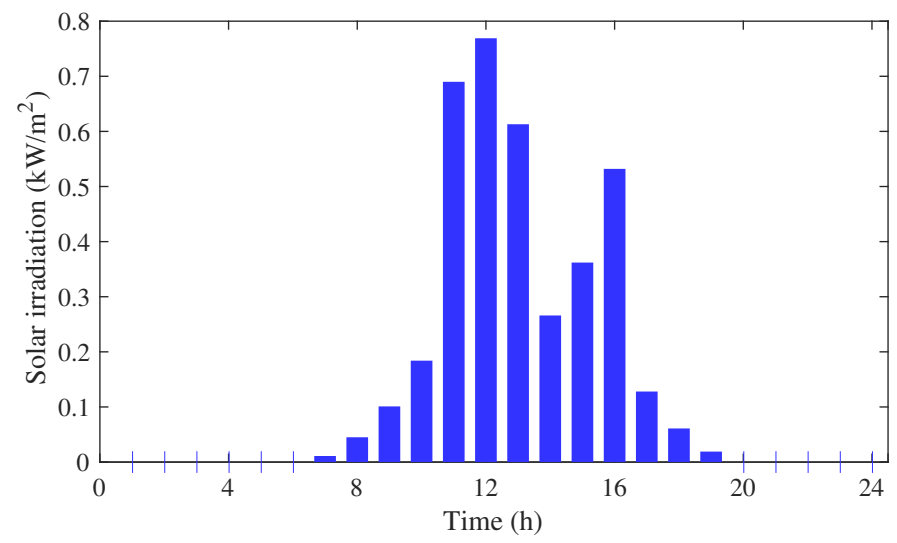

Figure 2: Predicted solar irradiation over a $24 \mathrm{~h}$ horizon.

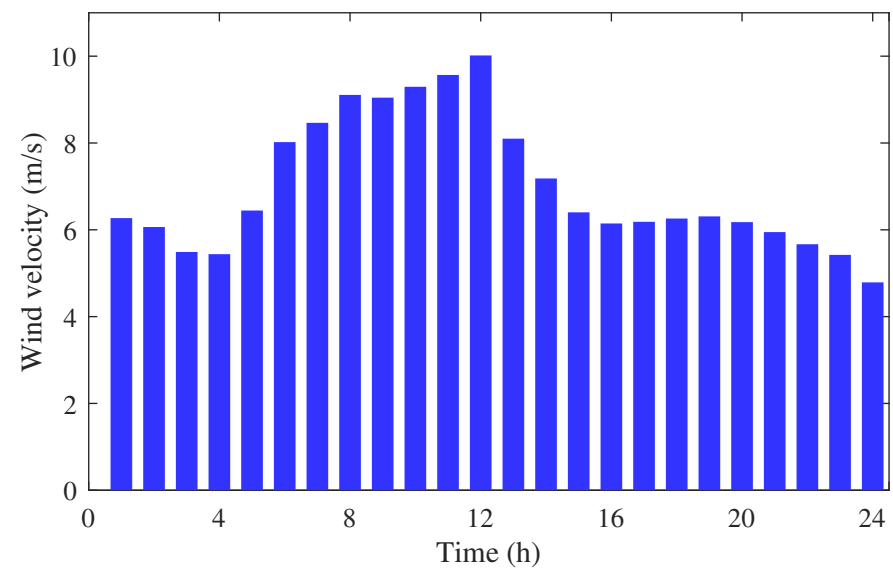

Figure 3: Predicted electricity prices over a $24 \mathrm{~h}$ horizon. 


\subsection{Wind turbine}

The electric power of a wind turbine that depends on wind velocities at the turbine power rating and site can be represented as a piece-wise function of the wind speed as follows [21]:

$$
P_{w}= \begin{cases}0 & \text { if } v_{f} \leq v \text { or } v \leq v_{c} \\ P_{r} \times \frac{v^{3}-v_{c}^{3}}{v_{r}^{3}-v_{c}^{3}} & \text { if } v_{c} \leq v \leq v_{r} \\ P_{r} & \text { if } v_{r} \leq v \leq v_{f}\end{cases}
$$

where $P_{r}$ and $v_{r}$ are the rated electrical power and the rated wind speed, respectively; $v$ and $v_{c}$ refer to wind speed and the cut-in wind speed, respectively; and $v_{f}$ the cut-off wind speed.

The total output power for a number of wind turbines can be extracted as follows:

$$
P_{w T}=P_{w} \times N_{w}
$$

where $N_{w}$ is the number of wind generators.

\subsection{Loads and utility grid}

The load profile of an individual house is illustrated in Figure 4, where the maximum load is around 2.2 $\mathrm{kW}$ that can cover the basic household equipment, such as lights, refrigerators, fans, TVs and computers. Residential load profiles, $L(t)$, are fluctuating with the time step of $1 \mathrm{~h}[15$. For simplicity, the load patterns are considered the same for all the households, even though different numerical results would be obtained using diverse loads for each customer, the conclusions that can be drawn from experiments remain unaltered.

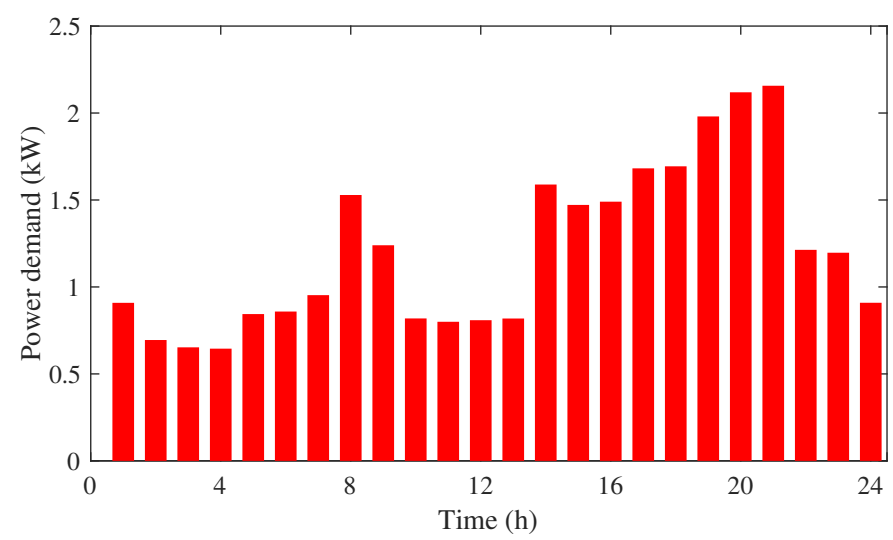

Figure 4: Predicted load profile over a $24 \mathrm{~h}$ horizon.

Real-time electricity price (RTP), an effective approach to manage power demand [22, is used to buy/sell electric power. The RTP is denoted as $C(t)($ cents/kW h) and is shown in Figure 5 . Buying and selling RTP, i.e. $C_{b u y}$ and $C_{\text {sell }}$, is considered the same for this analysis. The variation of RTP is taken from [23] and it is suitably modified for the future electricity market of Australia.

The exchange power at time $t$ with the grid utility is marked as $P_{g}(t) \mathrm{kW}$ and it can be calculated as follows:

$$
P_{g}(t)=L(t)-P_{s T}(t)-P_{w T}(t)+u(t)
$$

with the following conditions 


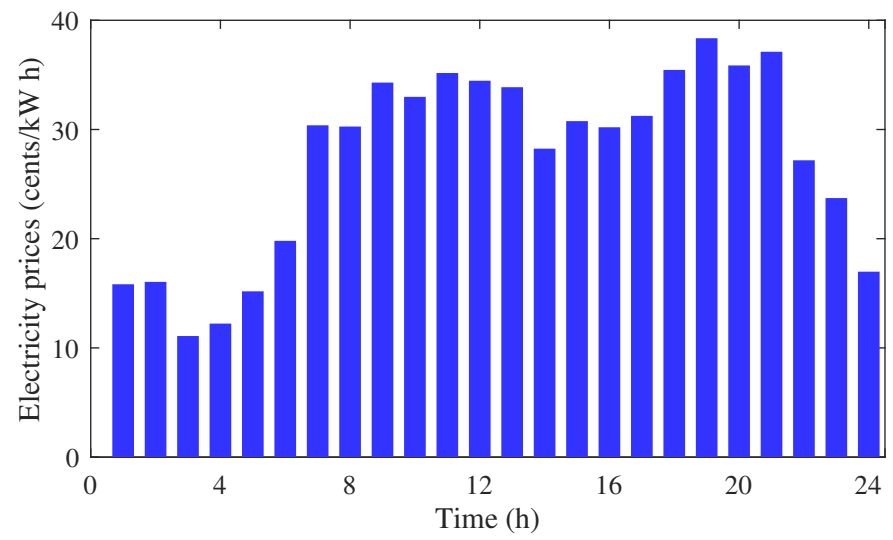

Figure 5: Predicted electricity prices over a $24 \mathrm{~h}$ horizon.

- $P_{g}(t)>0$ if power is imported from the grid, i.e., $P_{b u y}$ and

- $P_{g}(t)<0$ if power is exported to the grid, i.e., $P_{\text {sell }}$.

We have considered a community microgrid that is connected to the grid. It is assumed that the project is conducted in a place where the government encourages installing RESs for exchanging energy to the utility grid without any transmission limits. This is because of the lack of power generation, leading to low voltage profiles at the community level. As a result, local investors can invest in their own RESs to support the community as well as to make profits. In this case, the total operational costs can also be negative to indicate higher power generation than power demand, leading to financial benefits.

\subsection{Storage systems}

Although several forms of storage elements, such as flywheel, electrochemical battery and superconducting magnetic energy storage, are present in the market, lithium-ion (Li-ion) batteries are chosen in this study. They are one of the most popular types of storage systems and are increasingly being used in power system due to higher energy-to-weight ratios, a slow loss of charge during idle conditions and no memory effect [24, 25]. It is noteworthy that with different types of batteries, the results may vary slightly; however, the main purpose of this study remains the same, i.e., determining the actual operational cost of a microgrid.

The charging-discharging behaviours of the battery can be expressed with respect to battery energy levels as follows:

$$
\begin{array}{ll}
B L(t)=B L(t-1)+\Delta t P_{c}(t) \eta_{c} & \text { if the battery is charged } \\
B L(t)=B L(t-1)+\Delta t P_{d}(t) / \eta_{d} & \text { if the battery is discharged }
\end{array}
$$

subject to the following battery constraints:

Power limits

$$
\begin{aligned}
& P_{c, \text { max }}>P_{c}(t)>0 \\
& P_{d, \text { max }}<P_{d}(t)<0
\end{aligned}
$$

Battery energy level limits

$$
B L_{\max }>B L(t)>B L_{\min }
$$


where $P_{c}(t)$ and $P_{d}(t)$ are the charging powers of the battery at time $t$ and the discharging powers of the battery at time $t$, respectively; $B L(t)$ and $\Delta t$ are the battery energy level and the interval of the time period, respectively; and $\eta_{c}$ and $\eta_{d}$ are the charging and discharging efficiency, respectively. The $\eta_{c}$ and $\eta_{d}$ are considered as unity for simplicity.

\section{The proposed objective function}

The aim of this study is to minimise electricity cost in terms of energy exchange with the grid utility by controlling the battery energy efficiently while considering its degradation costs. The minimum cost to use electricity can be achieved mainly from two perspectives: demand-side management (controlling load) and generation side management with respect to power generated at the distribution level (effective and efficient use of exchanging power generated). In this paper, generation side management is emphasised to reduce the electricity costs of the community microgrid. To achieve minimum electricity costs, day-ahead scheduling is proposed with a new objective function which indicates the degradation costs of the energy storage system.

The minimum electricity costs can be achieved by purchasing energy during the low price periods and selling it at the time of high prices. One of the most cost effective formulations used in the literature [14, 15, 16, 19] for exchanging energy with the grid utility is represented as follows:

$$
F_{1}=\sum_{t=0}^{T} P_{b u y}(t) C_{b u y}(t)+\sum_{t=0}^{T} P_{\text {sell }}(t) C_{\text {sell }}(t)
$$

subject to the energy balanced equation

$$
P_{b u y}(t)+P_{s T}(t)+P_{w T}(t)=L(t)+u(t)+P_{\text {sell }}(t)
$$

where $C_{b u y}$ and $C_{\text {sell }}$ are the buying and selling electricity prices, respectively, and $P_{b u y}$ and $P_{\text {sell }}$ are the buying and selling power from and to the grid utility, respectively. The first part of Eq. (8) is the price of the power imported from the grid utility while the second one refers to selling power to the grid utility over the given time periods. As the objective function, Eq. [8], is directly related to power exchanges with the grid, it is used to optimise electricity costs for energy exchange program over the scheduling periods. However, this formulation ignores the degradation cost of a battery that has a substantial role in increasing the overall operational cost of the community microgrid, leading to lower profits.

In this paper, an equivalent degradation cost of charging-discharging cycles for a certain depth of discharge $(\mathrm{DoD})$ is proposed to the objective function as follows:

$$
F_{2}=\sum_{t=0}^{T} C_{t c y c} * L_{b}(t, d)
$$

where $C_{t c y c}$ is the cost involved for charging-discharging cycles from the total investment of the battery system and $L_{b}(t, d)$ is the life loss of the battery over the time period, $\mathrm{T}$, for counted cycles of depth, $d$, determined by applying the Rainflow algorithm.

The total life lost, $L_{b}$, from a SoC profile can be calculated by summing up the life loss of $N$ number of cycles as follows:

$$
L_{b}=\sum_{i} f_{c y c}\left(d_{i}\right)
$$




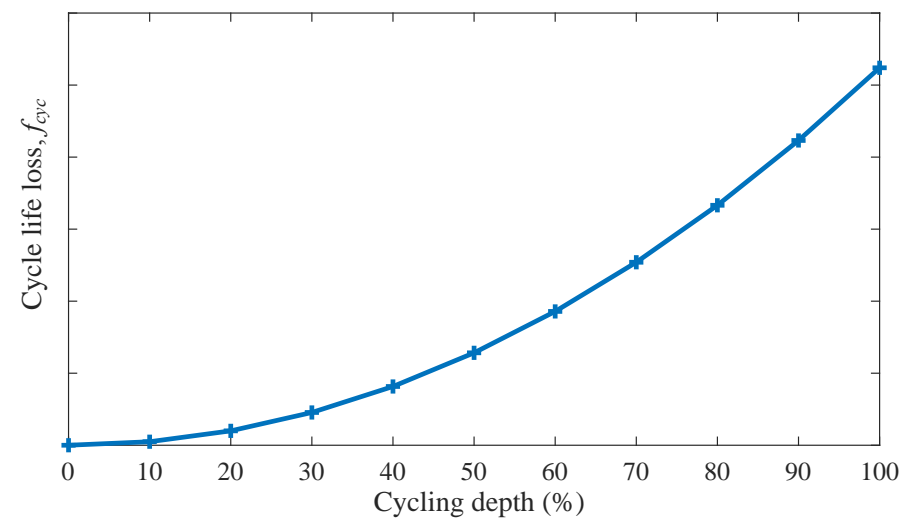

Figure 6: Typical life loss of a battery vs DoD.

where $f_{c y c}\left(d_{i}\right)$ is the cycle depth stress function of the battery for charging-discharging activities. It can be calculated as follows:

$$
f_{c y c}\left(d_{i}\right)=\frac{N_{c, i}}{\eta_{100}} d_{i}^{k_{p}} \quad i=1,2,3 \ldots
$$

where $N_{c, i}$ is the number of cycles with depth $d_{i}$, half and full, counted by applying the Rainflow algorithm, $d_{i}$ is the $i^{\text {th }}$ depth of the cycles' amplitude determined by the algorithm, $k_{p}$ is the slop value of the battery degradation curve taken from the battery sheets, and $\eta_{100}$ is the total number of given cycles for the battery. An exponential relationship between cycle life loss and depth of discharge typically exists as illustrated in Fig. 6.

The battery has generally two types of degradations: self (calendar fade) and cycle [26, 27,. Self degradation represents the corrosion of internal materials that has no relationship with charging-discharging cycles, and therefore is considered as a constant value, for example, if the self life of a battery is 10 years, then the degradation per day can be calculated as $\frac{100 \%}{365 * 10}=0.02739 \%$. In contrast, cycle degradation is related to the working modes of the battery [28, i.e., charging and discharging. For example, if the number of battery cycles with $80 \%$ DoD is 3000 , then each cycle counted from $100 \%$ to $20 \%$ of SoC reduces the battery life $\frac{100 \%}{3000}=0.033 \%$ of the total life. The cost related to the cycle degradation is taken into account in the objective function as it depends on the operational modes of the battery. If the self costs are taken out of the total cost, then cycling cost can be determined as follows:

$$
C_{t c y c}=C_{i n v}-C_{\mathrm{slf}}
$$

where $C_{i n v}$ indicates the total investment cost of the battery and $C_{\text {slf }}$ refers to the self degradation cost of the battery over the time period. $C_{i n v}$ includes the installation and maintenance costs of the battery, which involve purchasing the batteries, $B C$, that is proportional to the battery size, $B S(\$ / \mathrm{kWh})$. The costs of the battery can be represented as follows:

$$
C_{i n v}=B S \times M C+B S \times B C \times C R F
$$

where $M C$ is the maintenance costs expressed as $\$ / \mathrm{kW} \mathrm{h}$ and $C R F$ is a ratio for evaluating the current value of the battery with respect to the annual real interest rate, $r$, and the lifetime, $n$. $C R F$ can be expressed as follows:

$$
C R F=\frac{r(1+r)^{n}}{(1+r)^{n}-1} .
$$


Therefore, the objective function after including the degradation cost of the battery can be written as follows:

$$
\begin{aligned}
F & =F_{1}+F_{2} \\
& =\sum_{t=0}^{T} P_{b u y}(t) C_{b u y}(t)+\sum_{t=0}^{T} P_{\text {sell }}(t) C_{\text {sell }}(t)+\sum_{t=0}^{T} C_{t c y c} * L_{b}(t, d)
\end{aligned}
$$

where the first two parts of Eq. 16 is to make sure that the energy exchange with the grid utility is carried out with the lowest cost while the third part indicates the costs involved as a result of charging-discharging cycles. The minimum value of the objective function refers to the economical scheduling of the community microgrid while considering the operational cost of the battery.

\subsection{Constraints}

The following constraints are considered in the optimisation model for calculating a feasible solution of the cost functions.

Energy balance: The energy balance constraint, which indicates that the renewable energy sources, battery and grid utility should necessarily meet the power demand of the network at every hour, can be represented as follows:

$$
P_{b u y}(t)+P_{s T}(t)+P_{w T}(t)=L(t)+u(t)+P_{\text {sell }}(t) .
$$

As the positive values of $u$ indicate charging similar to a load characteristic; it is placed at the right-hand side of the equation.

Battery energy: The solution commands, $u(t)$, must fulfil the following constraints in order to extend the lifetime of a battery.

1) The charging and discharging rates of the battery must be within the prescribed limitations, i.e., $\left(P_{d, \max }<\right.$ $\left.u(t)<P_{c, \max }\right)$.

2) The energy level of the battery must not exceed the upper and lower limits at any time, i.e., $B L_{\min }<$ $B L(t)+u(t)<B L_{\max }$.

\section{Solution approaches}

In this research, the degradation cost of the battery and dynamic electricity prices are considered in the objective function, leading to complexity in solving it using conventional optimisation algorithms. To solve the function, a framework is designed based on two well-known algorithms such as (i) PSO and (ii) Rainflow. PSO is used to optimise the new proposed model while the Rainflow algorithm determines battery degradation costs during the search process. Both the PSO and Rainflow algorithms are discussed in subsections 5.1 and 5.2. respectively. The uncertainty of the renewable sources are represented in the form of different scenarios, in which each scenario represents a distinct input of the optimisation model. As previous research shows that a large number of scenarios are required to solve an uncertain optimisation problem [29, we use a reduction technique where a small number of effective scenarios are determined. Scenario generation is discussed in subsections 5.3 and its reduction technique is used as described in [30. The overall framework is discussed as follows.

Start: Initialisation 
1. Use the parameter values given in Table 1 .

2. Load the hourly data of wind speed, electricity price, solar irradiation and load profile. In case of uncertainty consideration in RESs and RTP, the data are taken from a scenario generated in subsection 5.3 and repeat same process for all considered scenarios;

3. Compute total solar power, wind power and loads;

4. Start PSO algorithm;

5. Initialise the position and velocity vectors for all particles randomly with values between upper and lower limits;

6. Determine the energy levels of the battery over the time period;

7. Apply the Rainflow algorithm as described in subsection 5.2 to determine the total number of cycles and their amplitudes;

8. Estimate the total damage to the battery;

9. Evaluate the cost function in Eq. 16 to find fitness value for the scheduling time;

10. Run the algorithm (iteration)

(a) Initialise positions and velocities again after the first run;

(b) Modify the velocities and positions of all particles according to Eqs. 20) and (19), respectively;

(c) Calculate available battery energy for the forecasting horizon, freeze the solutions if the battery constraints are violated by imposing limits;

(d) Repeat the procedure (7)-(9);

(e) Synthesise the global best and individual best values by comparing costs;

11. Continue the steps from 10 until the termination criterion is fulfilled.

\subsection{PSO algorithm}

PSO is a population-based optimisation method that is inspired by the social behaviour of fish schooling or birds flocking [31. It shares many similar features with evolutionary algorithms, such as genetic algorithm, and is effectively employed to solve many power system problems [32, 33. An overview of PSO in the application of power system with its advantages over other optimisation algorithms and fast convergence rate is discussed in 34 .

Two equations for the position and velocity vectors in an $N$-dimensional solution space are the most important aspect of the PSO [35. The movement and position of each particle $i$ can be represented as $v_{i}^{k+1}$ and $x_{i}^{k+1}$ vectors, respectively, as follows:

$$
\begin{aligned}
& v_{i}^{k+1}=w v_{i}^{k}+c_{1} r_{1}\left(p_{i}^{k}-x_{i}^{k}\right)+c_{2} r_{2}\left(p_{g}^{k}-x_{i}^{k}\right) \\
& x_{i}^{k+1}=x_{i}^{k}+v_{i}^{k+1}
\end{aligned}
$$


where $v_{i}^{k}$ and $x_{i}^{k}$ refer to the $i^{\text {th }}$ particle velocity for $k^{\text {th }}$ iteration in $N$-dimension and to the $i^{\text {th }}$ particle position for $k^{\text {th }}$ iteration in $N$-dimension, respectively; $p_{i}$ and $p_{g}$ are the best position of an individual particle $i$ and the best position achieved among all particles, respectively. In addition, $r_{1}, r_{2}$ represent the random numbers of the uniform distribution within the range of [0 1$]$, and $c_{1}, c_{2}$ are the learning factors used to control the significance of the best solution. Generally, population size is set in the range of 20-50, and the values of $w$ and $c_{1}, c_{2}$ are considered as one and two, respectively.

The constriction coefficients, used for restricting the particle velocity to avoid unacceptable velocity within the next few iterations, are proposed in [36]. The coefficient controls the particle movements and guides them toward the convergence. The modified velocities of particles can be represented as follows:

$$
v_{i}^{k+1}=w_{C F}^{k} v_{i}^{k}+C_{1} r_{1}\left(p_{i}^{k}-x_{i}^{k}\right)+C_{2} r_{2}\left(p_{g}^{k}-x_{i}^{k}\right)
$$

where $w_{C F}=w \times C F$, and $C_{1}, C_{2}$ refer to the cognitive and social components, respectively. They have an effect on searching the optimal point in the given solution space and convergence rate of the algorithm. The $C_{1}$ and $C_{2}$ can be expressed as follows:

$$
\begin{aligned}
& C_{1}=C F \times \phi_{1} \\
& C_{2}=C F \times \phi_{2}
\end{aligned}
$$

where

$$
\begin{array}{r}
C F=\frac{2}{\left|\phi-2+\sqrt{\phi^{2}-4 \phi}\right|} \\
\phi=\phi_{1}+\phi_{2} \\
\phi_{1}+\phi_{2} \geq 4
\end{array}
$$

where $C F$ and $\phi$ represent a constriction factor and co-efficient, respectively. Although the constriction factor can assist in converging the optimisation process toward optimal solutions, a better approach is to set limits of positions and velocities.

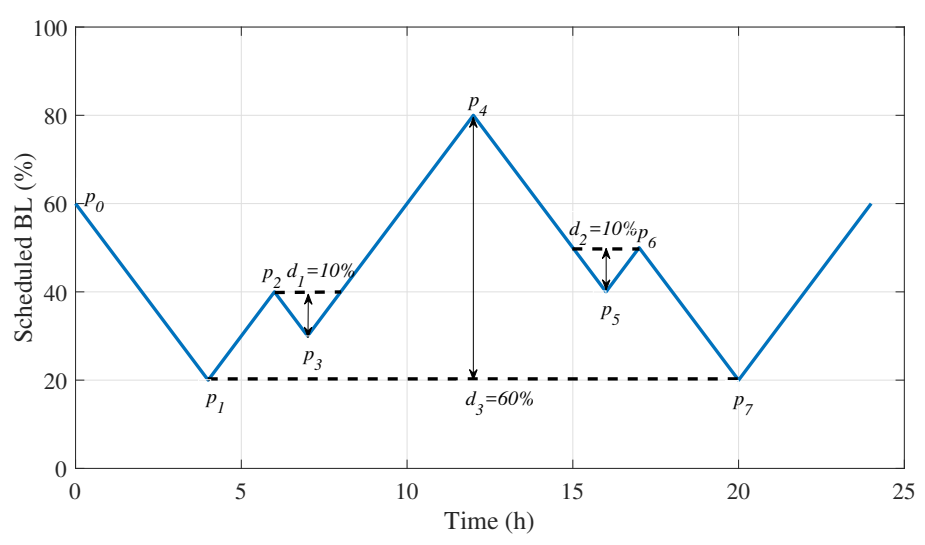

Figure 7: Cycles counting process by the Rainflow algorithm.

\subsection{Rainflow algorithm}

To determine charging-discharging cycles and measure their cumulative effect, the Rainflow counting algorithm is used in this paper. The algorithm counts cycles and measures their amplitudes for a given SoC profile with a series of local extrema as follows [37, 7]. 
1. Start counting and measuring from the beginning of the battery energy level (BL) as shown in Figure 7

2. Calculate the difference between two turning points as follows: $\Delta p_{1}=\left|p_{1}-p_{0}\right|, \Delta p_{2}=\left|p_{2}-p_{1}\right|$, $\Delta p_{3}=\left|p_{3}-p_{2}\right|$, and so on.

3. A full cycle of depth $\Delta p_{2}$ is identified if $\Delta p_{2} \leq \Delta p_{1}$ and $\Delta p_{2} \leq \Delta p_{3}$. Eliminate the points $\left(p_{1}\right.$ and $\left.p_{2}\right)$ associated with the cycle from the profile, and then repeat the process using other points $p_{0}, p_{3}, p_{4}, p_{5} \ldots$

4. If a cycle is not identified, shift the searching points forward and repeat the process using points $p_{1}, p_{2}, p_{3}, p_{4}$ and so on.

5. The cycle detection process is repeated until no further full cycles are identified throughout the remaining profile.

The rest of energy profile after detecting full cycles contains half cycles that are linked to each other by their neighbouring local extrema in the profile. A simple example of counting the SoC profile depicted in Figure 7 has two full cycles of depth $10 \%$ and one full cycle of depth $60 \%$, as well as a discharging half cycle of depth $40 \%$ and charging half cycle of depth $40 \%$.

\subsection{Scenario generation}

As the power output of RESs are stochastic in nature and thus RTP is set by the network operators hourahead, solutions of the problem formulated may not work effectively based on the prediction values. To see the effect of uncertainty on the optimisation problem, we have represented these variables in the form of random scenarios. Scenarios with a normal distribution (Nor) over the time period (T) are generated based on their mean values $(\mu)$ that depend on forecasting values and standard deviation $(\sigma)$, which generally depends on the historical errors [38. The scenario generation procedures are described as follows:

1. Set predicted values as mean values

2. Select standard deviation for input data

3. Set the number of scenarios (ns)

4. Start Iteration

(a) Generate a number of random variables using normal distribution, as: $\mathrm{Y}_{t, s}=$ Nor $\left(\mu_{t}, \sigma_{t}\right), \quad \mathrm{s}=1,2$, $3 \ldots$ ns and $\mathrm{t}=1,2,3 \ldots \mathrm{T}$

5. Continue the process from 4 until the termination criterion, i.e. time horizon $(\mathrm{T})$, is fulfilled

\section{Simulation results}

In this section, simulation is carried out to demonstrate the effectiveness of the proposed cost function by applying various scenarios. The community microgrid connected to the grid utility maintains a balanced power supply and demand relationship with the help of the EMS, taking into consideration the need to provide minimum electricity costs to the community. As power generated by solar and wind generators is fully utilised to maximise profits using a storage system, an efficient scheduling of the battery energy with respect to RTP 
Table 2: Statistical analysis of four cases for 31 runs.

\begin{tabular}{|cccccc|}
\hline Cost function & Min & Max & Average & STD & Median (cents) \\
\hline EOFP & 1042.03 & 1133.50 & 1099.67 & 21.42 & 1102.77 \\
EOFR & 1033.62 & 1062.68 & 1040.39 & 5.70 & 1038.42 \\
POFP & 1054.33 & 1094.67 & 1070.71 & 9.77 & 1068.55 \\
POFR & 1046.70 & 1054.95 & 1049.36 & 2.40 & 1050.30 \\
\hline
\end{tabular}

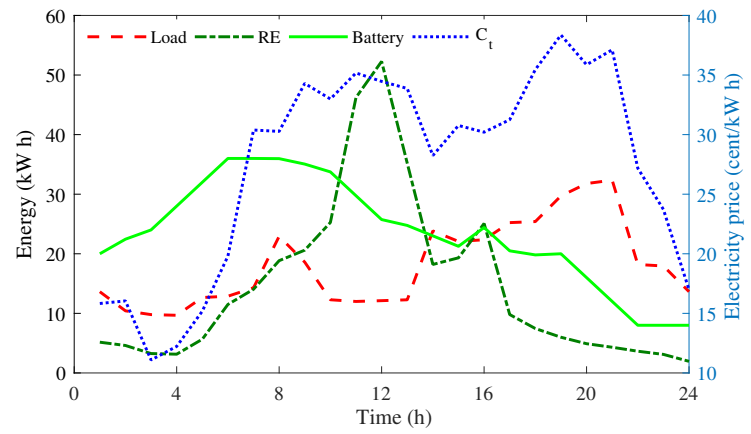

(a)

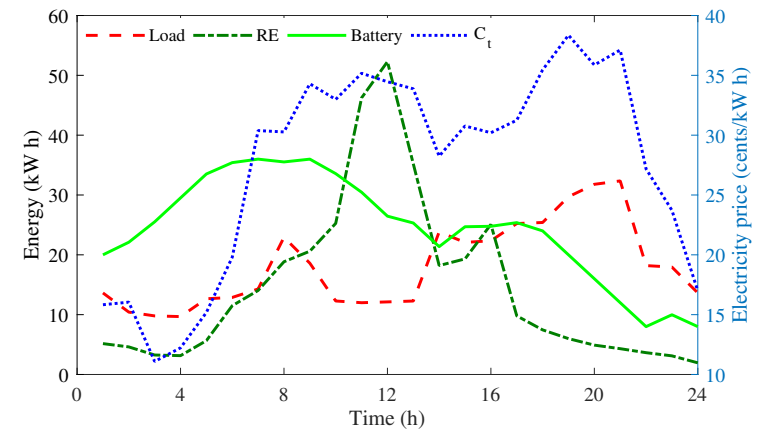

(c)

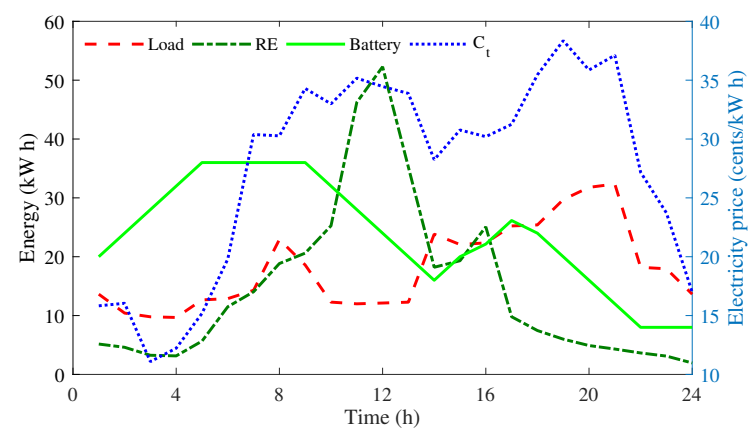

(b)

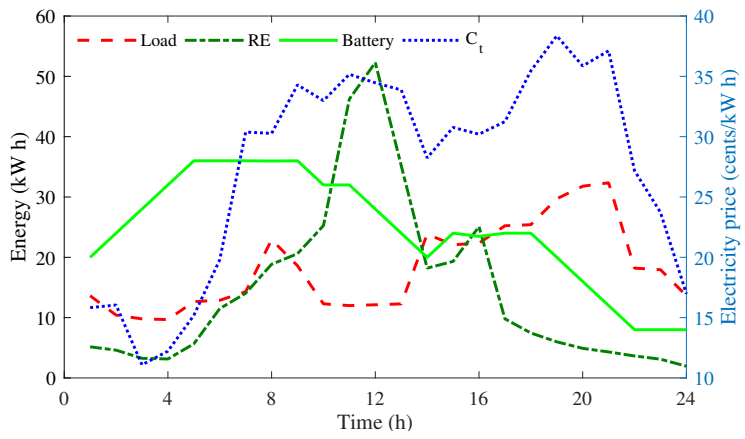

(d)

Figure 8: Energy scheduling of the microgrid for four cases: (a) EOFP (b) EOFR (c) POFP and (d) POFR.

is the primary goal of this study. The parameters used in this study are shown in Table 1. It is worth mentioning that $40 \mathrm{kWh}$ battery size is chosen to meet the maximum power demand for one hour if the battery constraints are neglected. It is assumed that using this small capacity of the storage system for energy management of the community microgrid is more difficult due to its limited storage capacity than a large storage system. The simulation is carried out for a day-ahead scheduling program, with different scenarios to demonstrate the effectiveness of the proposed cost function. The new model (dimension size is 24) is solved using the modified PSO algorithm. After several parametric tests, the population size, maximum number of generation, constriction co-efficient and inertia coefficient are considered as 30, 1000, 4.1 and 0.7, respectively. The algorithm is implemented on a personal computer with a 3.4-GHz Intel Core i7 processor with 16 GB of RAM using the MATLAB (R2018a) environment. 


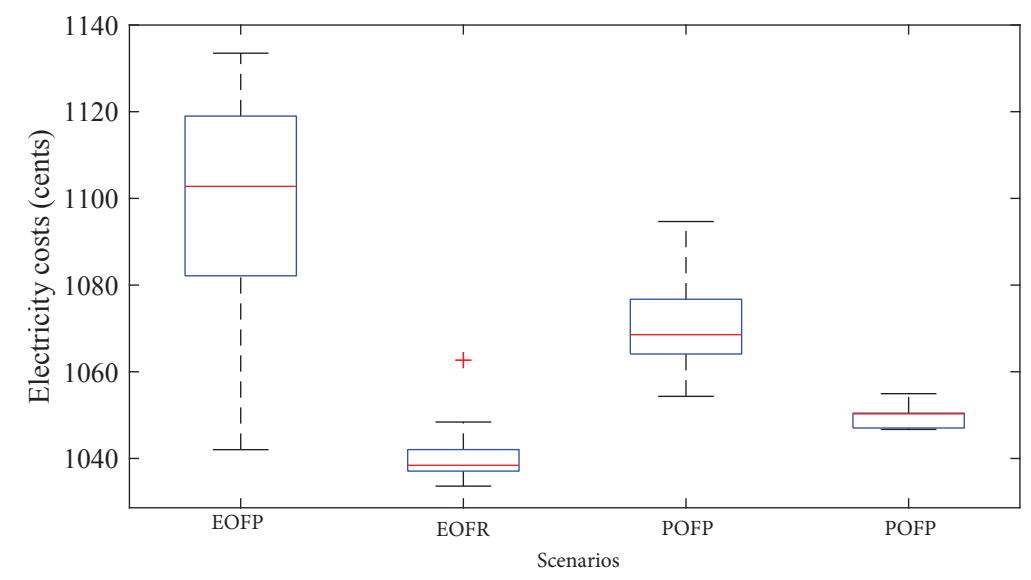

Figure 9: Box plot of four operational cases for 31 runs.

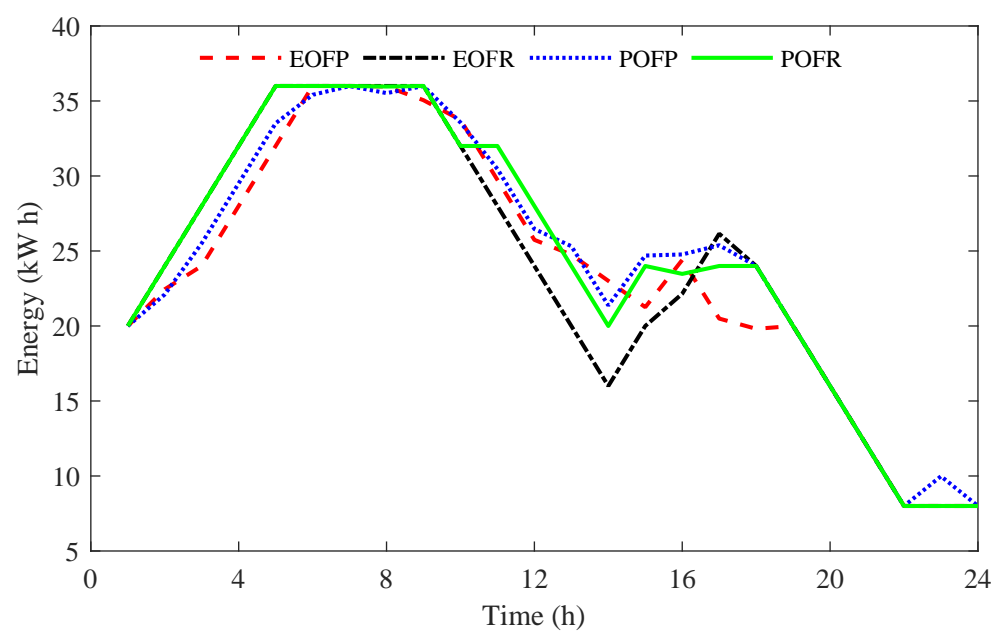

Figure 10: Comparisons of battery commands.

\subsection{Case studies}

\subsubsection{Energy management operation}

The aim of this study is to determine the actual operational costs of a microgrid through analysing existing and proposed objective functions in different forms, namely four cases. These case studies help to figure out the best objective function among the existing and proposed ones for the energy management of the community microgrid with actual operational costs. As there are four possible ways to evaluate the two objective functions- existing and proposed, we have formulated the objective function as follows: existing objective function with penalty method (EOFP), existing objective function with repairing method (EOFR), proposed objective function with penalty method (POFP) and proposed objective function with repairing method (POFR). The cost function $F_{1}$ in Eq. $(8)$ is used as an existing objective function, whereas $F$ in Eq. 16 is the one proposed in this study. It is worth noting that the penalty method, most commonly used one [18. 19, 13], discards the solution vectors when constraints are violated, whereas the repairing method sets threshold points for not satisfying the constraints. It is expected that EOFR should have lower electricity costs than other cases since it has no degradation costs of the battery but rather is equipped with repairing methods. In the existing objective function, power exchange with the grid is carried out without considering degradation costs of a battery while the proposed one takes into account the costs of the battery. 
Table 3: Actual cost determination of four cases.

\begin{tabular}{|ccccc|}
\hline Cost function & Degradation costs & Median operational cost & Total costs (cents) & ETOF $(\mathrm{h})$ \\
\hline EOFP & 200.35 & 1102.77 & 1303.12 & $1.189 \times 10^{9}$ \\
EOFR & 241.71 & 1038.42 & 1280.13 & $9.858 \times 10^{8}$ \\
POFP & 206.29 & 1068.55 & 1274.84 & $1.155 \times 10^{9}$ \\
POFR & 203.73 & 1050.30 & 1254.03 & $1.169 \times 10^{9}$ \\
\hline
\end{tabular}

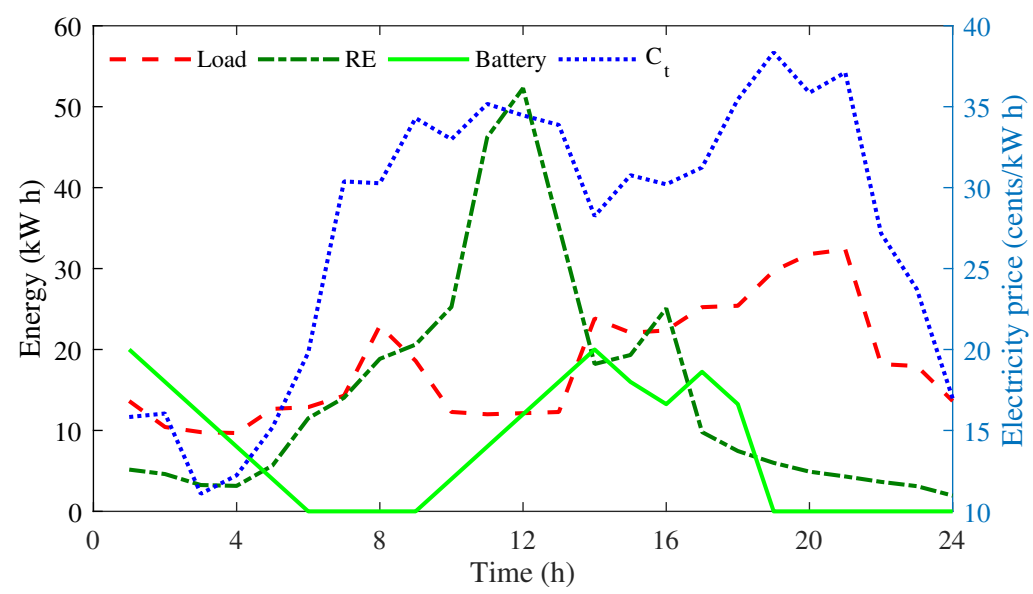

Figure 11: Baseline approach for the microgrid operation.

A day-ahead scheduling approach for the microgrid is carried out to demonstrate a comparison. Parameters for the experiments are taken from real measurements of data but with reduced scale to match the community microgrid. As the algorithms work on random variables, we have conducted the experiment for thirty-one runs to statistically demonstrate the results. Each run takes a different seed value for generating initial variables. A statistical comparison chart of the four cases is tabulated in Table 2 in which it is shown that EOFR has the lowest value (1033.62 cents) among the scenarios while POFP posses the highest value (1054.33 cents) after completing the predetermined runs. The reason is that EOFR has used the repairing method that provides better solution variables due to not discarding some competitive solutions while violating the constraints, and it has not considered a battery degradation cost that adds value to the objective function. In contrast, POFP has a degradation cost and it has also a drawback of discarding the solutions that violate constraints instead

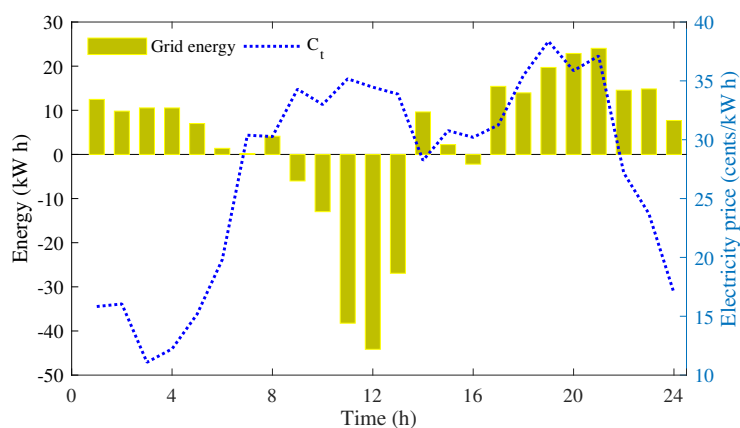

(a)

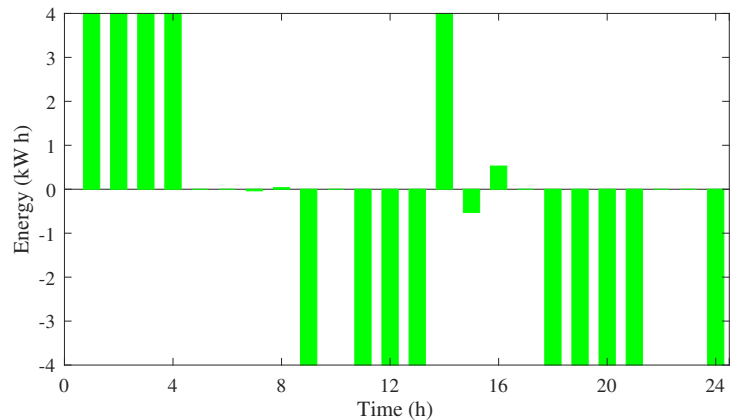

(b)

Figure 12: POFR energy scheduling: (a) energy exchange with grid and (b) battery commands. 


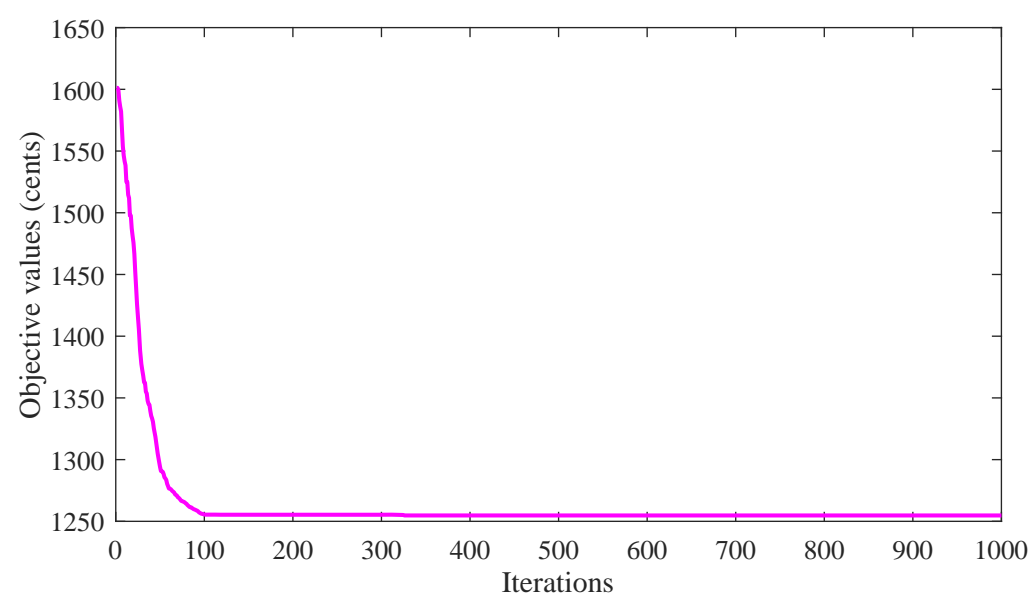

Figure 13: Convergence curve of the PSO algorithm.

of repairing them. From Table 2, it can also be observed that the proposed method, POFR, has the lowest standard deviation among the cases, i.e., whenever the algorithm is run, it has less deviated solutions among the approaches. The highest deviation is seen in EOFP as this approach discards the solutions for not satisfying the constraints.

The median values of the scenarios are taken into account to assess the comparisons as depicted in Figures 8. 9 and 10. It is worth pointing out that the energy and power units have been used on one scale $(\mathrm{kW} \mathrm{h})$ as the one-hour time interval (sampling time) is considered. It is evident from Figure 10 that the patterns of charging and discharging are similar and it can be apparently assumed that EOFR is the best solution for the operation as it has the least operational median cost as shown in Table 2 However, an analysis after determining degradation costs of the charging-discharging profiles with the help of the Rainflow counting algorithm recommends the proposed function, i.e. POFR, which has the least operational cost all together (degradation costs + operational costs) as demonstrated in Table 3 It is also observed that POFP has the second least operational cost that refer to the superiority of the proposed objective function to the existing ones. Although EOFP has the least degradation costs with increased lifetime, i.e., expected time to operational failure (ETOF), it has the highest true operational cost that overpowers the degradation cost. Therefore, it can be concluded that the proposed method, POFR, has the highest cost saving compared to the other methods.

\subsubsection{Comparing the operation with baseline algorithm}

The proposed method is compared with a baseline algorithm to demonstrate the effectiveness of implementing the optimisation algorithm with the problem formulated. The rules of the baseline algorithm are described as follows [13]:

- If load demand is greater than power generation, then power demand is supplied from the battery maintaining the battery constraints. If the battery cannot supply the required power, then import the power from the grid utility.

- If power generation is higher than power demand, then extra power is used to charge the battery with the maintenance of the charging rate. If the surplus power is greater than the charging rate or the battery is already full, the remained energy is exported to the grid. 
The simulation results shown in Figure 11 indicate that whenever renewable power generation is lower than power demand, the battery discharges, and it charges during higher power generation than power demand. This procedure can be simply implemented by if-else statements. The operation costs of this method over the time period is 2077.14 cents. On the other hand, the result of the proposed method is illustrated in Figure $8 \mathrm{~d}$. In this method, charging-discharging behaviours follow a pattern of electricity prices as formulated in Eq. 16 considering battery degradation costs. It is observed that the battery charges when electricity prices are low and it discharges during higher electricity prices. This leads to operational costs 1254.03 cents, which is $39.63 \%$ less than the baseline algorithm. The energy scheduling program with the grid utility and the battery commands for the charging-discharging behaviours is depicted in Figure 12 . The positive values indicate imported power from the grid during the shortage of renewable power, while the negative values refer to the exported power to the grid. The convergence of the PSO algorithm is illustrated in Figure 13.

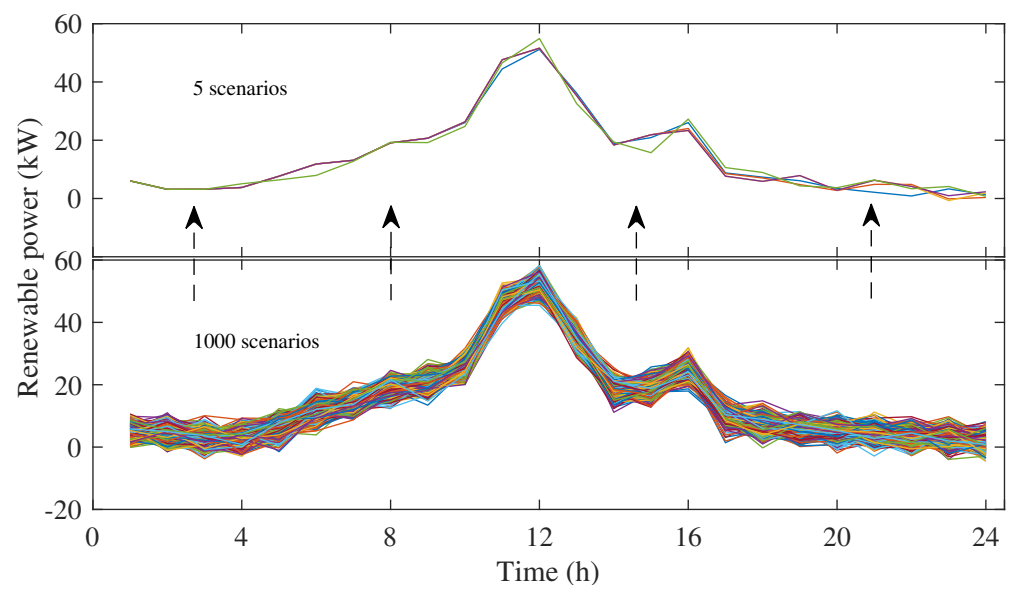

Figure 14: Scenarios of renewable power.

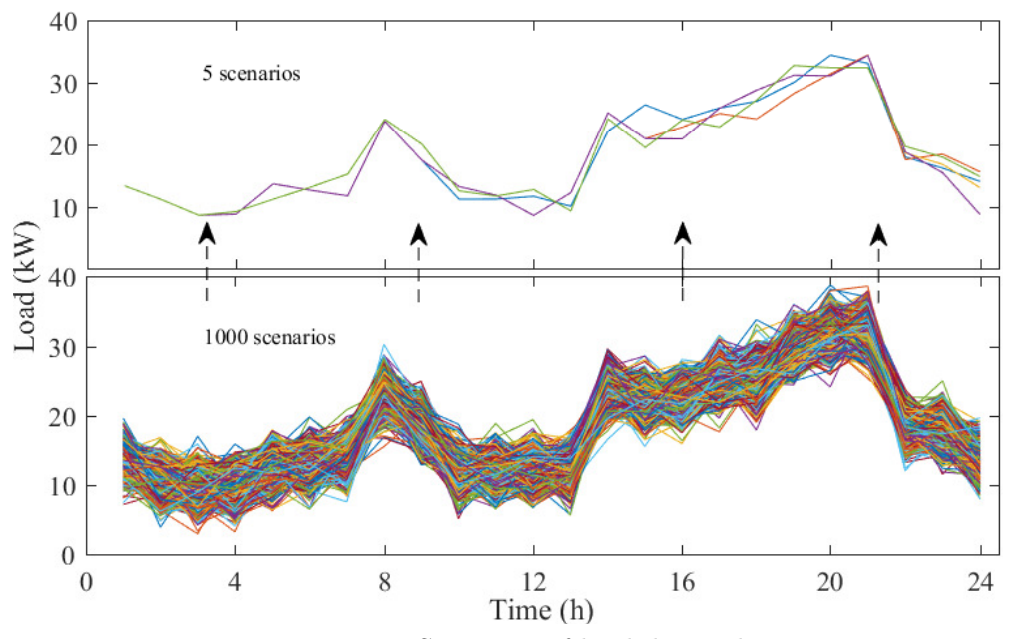

Figure 15: Scenarios of load demand.

\subsubsection{Impact of uncertainty in energy management}

The aim of this study is to analyse the effect of uncertainty in the scheduling program of the community microgrid. Uncertainty can appear in the scheduling program from various ways, such as intermittent power generation, the power demand of the community and real-time electricity prices. To demonstrate its effect on 


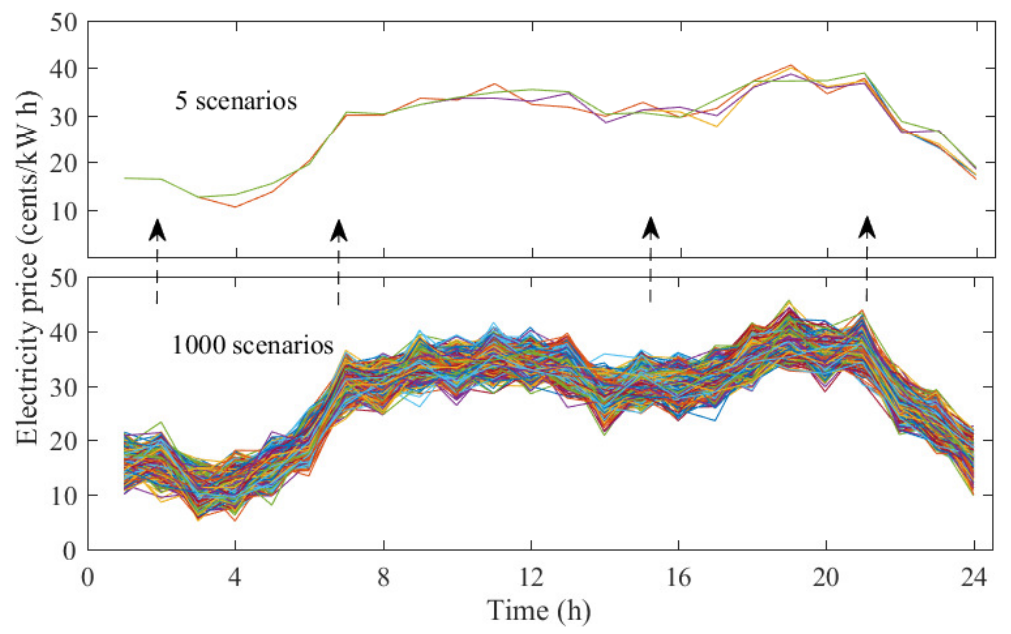

Figure 16: Scenarios of electricity price.

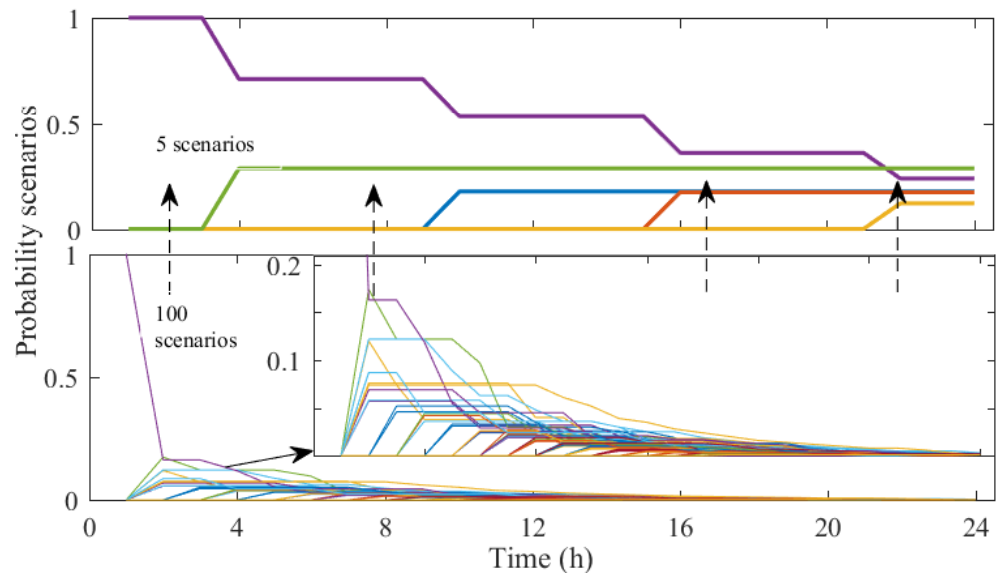

Figure 17: Probability of scenarios.

the scheduling, different scenarios of power generation, power demand and RTP are generated as described in subsection 5.3 using a standard deviation of 2 around the predicted inputs to capture a wider uncertainty band. Initially, one thousand scenarios are generated and then these are reduced to five scenarios using scenario reduction techniques described in [30]. The reduced scenarios for renewable power, power demand and electricity prices are shown in Figures 14, 15 and 16, respectively. Transformation of their probability from one thousand to five scenarios is also depicted in Figure 17. While five scenarios are extracted by the reduction technique, another three scenarios are also added to increase the versatility of input uncertainties as shown in Figures 18 19 and 20. Eight scenarios (S1-S8) in total for each input (generations, loads or electricity prices) are used to evaluate the effect of uncertainty. The first scenario is from input values; scenarios, S2 - S6, are from reduced scenario techniques; S7 from four hours behind the input profile and S8 from four hours ahead the input profile. These scenarios are marked as Load $1-8$, Renew $1-8$ and $\mathrm{C}_{\mathrm{t}} 1-8$. It is seen from Figures 14 , 15 and 16 that the levels of uncertainties during the first period are the same for the five scenarios but deviations increase among them with increasing time horizon since there is a high possibility to produce higher prediction error at the end periods, i.e., $\mathrm{t}=24 \mathrm{~h}$.

From Figures 18 and 19 , it is observed that there are no significant changes in the output of the battery commands although high uncertainty exists in the both power generation and power demand. In contrast, from 


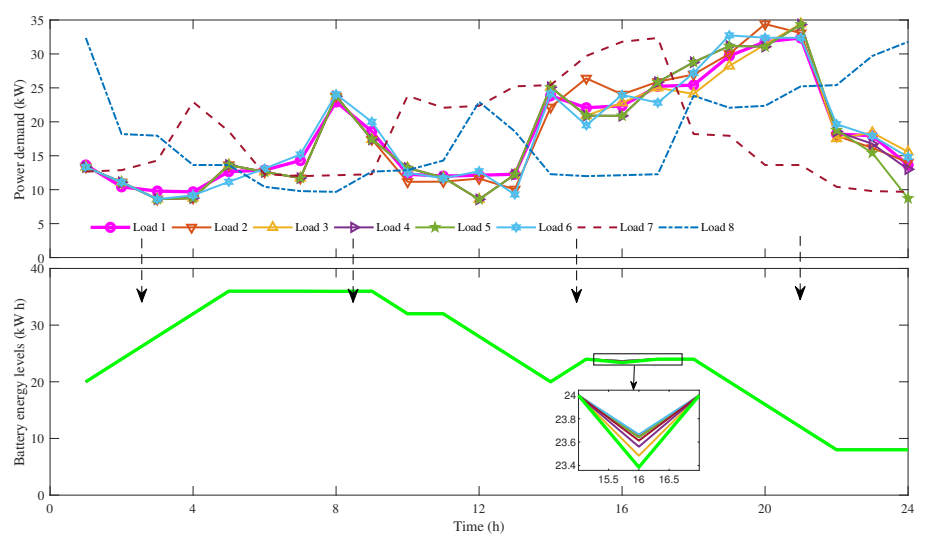

Figure 18: Effect of different load profiles on the outcome.

Figure 20, it can be seen that there is a significant influence of the battery commands for changing RTP. This is because of the direct influence of the electricity prices on the outcome. In this analysis, different operational costs are calculated, namely electricity costs with the renewable sources and battery storage (Cost 1), electricity costs with the renewable sources but without the battery storage (Cost 2) and electricity costs without the renewable sources and battery storage (Cost 3). For different patterns of renewable power generation, it is observed from Table 4 that electricity costs can be saved on average by $90.13 \%$ with respect to Cost 3 and $41.95 \%$ with respect to Cost 2. It is also noticeable that Cost 3 is constant throughout the scenarios due to the fact that power consumption have no relationship in varying renewable power generation. Similarly, average electricity cost saving for various load scenarios shown in Table 5 is $93.48 \%$ with respect to Cost 3 and $54.88 \%$ with respect to Cost 2 . For various electricity price schemes, the average electricity cost saving from Table 6 is $91.17 \%$ with respect to Cost 3 and $45.46 \%$ with respect to Cost 2 . It is apparent that using renewable power generation with an ESS can be a profitable investment, where controlling power is in the owners' hands. This analysis also reveals that forecasting errors in determining electricity price can lead to higher operational costs whereas errors in power generation and demand have no influence on the estimated costs.

\subsection{Algorithm comparisons}

This section is dedicated to comparing the PSO algorithm with the recently developed algorithms, namely flower pollination algorithm (FPA) [39, ant lion optimizer (ALO) [40, genetic algorithm (GA) [1], novel bat (NBAT) algorithm [42] and firefly algorithm [4].

To bring all the algorithms to the same platform, the number of population size and the maximum number of generations are set as thirty and one thousand, respectively, for all the algorithms. Although the parameters of the algorithms are tuned in some cases after several parametric tests, mostly the default values are found effective. As the algorithms take decisions randomly, we run thirty-one times to statistically demonstrate the effectiveness of the algorithm presented. Comparisons shown in Figure 21 and Table 7 are carried out using EOFP as this is a well-established formula used in the literature. The minimum electricity cost of the algorithms is 1042.03 cents that are obtained by the PSO algorithm followed by GA, whereas ALO algorithm exhibits the highest deviation. A Wilcoxon test, where P-values less than 0.05 indicate the statistical significance of the PSO algorithm [29], is also conducted for the statistical comparison purposes and it is observed that PSO has higher statistical significance than all other algorithms used as shown in Table 8 . Therefore, it can be concluded 
that the PSO algorithm has demonstrated superior performance to the other optimisation algorithms.

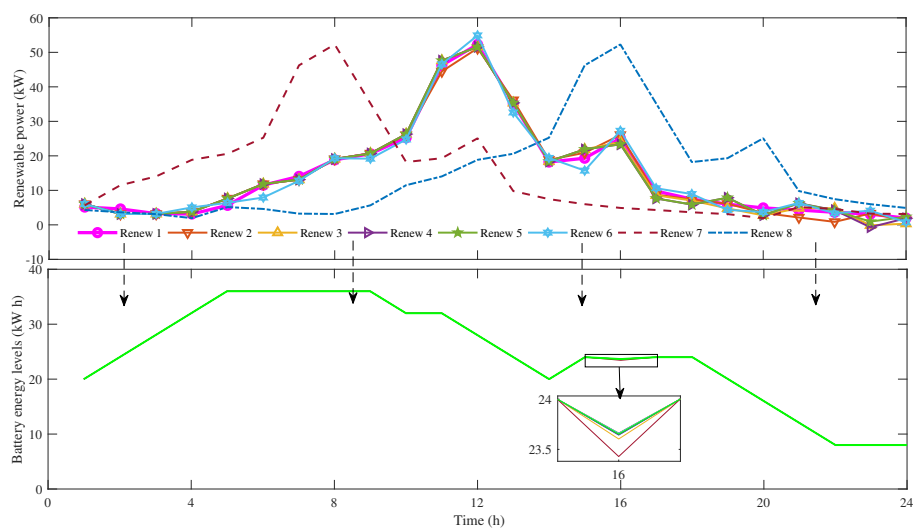

Figure 19: Effect of different power generations on the outcome.

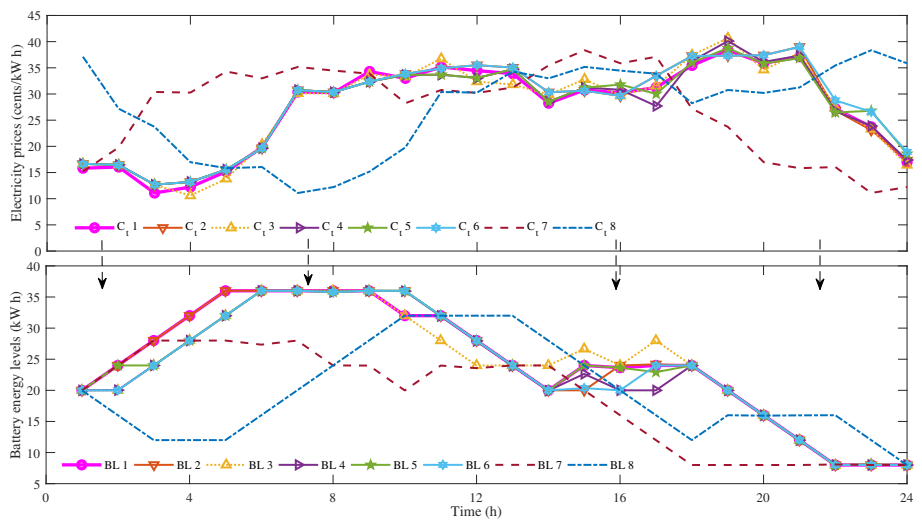

Figure 20: Effect of different electricity prices on the outcome.

\subsection{Discussion}

We have conducted a number of experiments for different case studies to validate the effectiveness of the proposed model to the optimisation problem. The key findings of the study are demonstrated in 'Simulation results' section and they can be summarised as follows.

Firstly, a novel way of modelling the battery operational costs based on the depth of charge-discharge patterns is proposed. The cost is included in the objective function to reflect the true operational cost of a microgrid. To determine the degradation cost, the Rainflow algorithm is integrated into the framework developed for solving the optimisation problem. The simulation results demonstrate that including degradation cost reveals the actual operational cost of the microgrid. The proposed degradation model can easily be adopted for real application to all the energy management approaches and also to participate in electricity markets, where battery involvement is present.

Secondly, uncertainties in power generation, power demand and electricity prices are investigated to observe their effects on the energy management of the microgrid. From the analysis, it is found that uncertainty in power generation and power demand has no significant influence on the scheduling energy, but RTP has an impact. These results are based on the assumption of unlimited energy exchange with the grid utility, but they may vary if constraints on energy exchange are imposed in the optimisation problem or if there are other generators, 
Table 4: Stochastic effects of generations on operational costs.

\begin{tabular}{|cccc|}
\hline Scenarios & Cost 1 (\% Saving w.r.t 3/2) & Cost $2(\%$ Saving w.r.t. $3 / 2)$ & Cost $3(\%$ Saving w.r.t. 3/2) \\
\hline Renew 1 & $1050.40(91.82 / 45.38)$ & $1923.18(85.01 / 0)$ & $12833.26(0 /-)$ \\
Renew 2 & $1225.79(90.45 / 41.59)$ & $2098.57(83.65 / 0)$ & $12833.26(0 /-)$ \\
Renew 3 & $1146.80(91.06 / 43.22)$ & $2019.61(84.26 / 0)$ & $12833.26(0 /-)$ \\
Renew 4 & $1064.94(91.70 / 45.04)$ & $1937.72(84.90 / 0)$ & $12833.26(0 /-)$ \\
Renew 5 & $1022.53(92.03 / 46.05)$ & $1895.31(85.23 / 0)$ & $12833.26(0 /-)$ \\
Renew 6 & $1144.30(91.08 / 43.27)$ & $2017.07(84.28 / 0)$ & $12833.26(0 /-)$ \\
Renew 7 & $2371.99(81.52 / 26.90)$ & $3244.90(74.71 / 0)$ & $12833.26(0 /-)$ \\
Renew 8 & $1103.77(91.40 / 44.16)$ & $1976.55(84.60 / 0)$ & $12833.26(0 /-)$ \\
\hline
\end{tabular}

Table 5: Stochastic effects of load profiles on operational costs.

\begin{tabular}{|cccc|}
\hline Scenarios & Cost $1(\%$ Saving w.r.t. $3 / 2)$ & Cost $2(\%$ Saving w.r.t. $3 / 2)$ & Cost $3(\%$ Saving w.r.t. 3/2) \\
\hline Load 1 & $1050.40(91.82 / 45.38)$ & $1923.18(85.01 / 0)$ & $12833.26(0 /-)$ \\
Load 2 & $1092.37(91.52 / 44.41)$ & $1965.14(84.74 / 0)$ & $12875.22(0 /-)$ \\
Load 3 & $839.53(93.35 / 50.97)$ & $1712.41(86.43 / 0)$ & $12622.48(0 /-)$ \\
Load 4 & $1018.17(92.05 / 46.16)$ & $1891.01(85.23 / 0)$ & $12801.09(0 /-)$ \\
Load 5 & $918.73(92.77 / 48.72)$ & $1791.52(85.90 / 0)$ & $12701.60(0 /-)$ \\
Load 6 & $1206.31(90.71 / 41.98)$ & $2079.08(83.99 / 0)$ & $12989.16(0 /-)$ \\
Load 7 & $540.16(95.62 / 61.77)$ & $1412.97(88.53 / 0)$ & $12323.05(0 /-)$ \\
Load 8 & $3.41(99.97 / 99.61)$ & $876.34(92.56 / 0)$ & $11786.42(0 /-)$ \\
\hline
\end{tabular}

such as fuel cells and micro-turbines. Although different scenarios for uncertainty are generated using normal probability distributions, no other solution approach except the one proposed here is used to minimise their effect on outcome. The reason is that the mean of the normal distributions for generating scenarios is the predicted data. The forecasting data have thus already taken the best positions among all the data in the normal distributions. This concludes that no solution approach except the one presented here is needed to minimise the uncertainties of normal distributions. In our analysis, it is observed that the microgrid can save on average $91.57 \%$ of the operational costs as compared to the only grid-connected distribution network, without RESs and an ESS. In addition, it can reduce costs on average by $47.43 \%$ as compared to the distribution network possessing only RESs. These high amounts of saving are due to the same RTP used for buying and selling energy, but these prices may not be the same in practice. If the selling prices are less than buying prices, the microgrid can still save a significant amount of electricity costs. It is worth noting that the percentage saving can even increase beyond $100 \%$, indicating a profit rather than a saving for operating a microgrid with the grid. 
Table 6: Stochastic effects of electricity prices on operational costs.

\begin{tabular}{|cccc|}
\hline Scenarios & Cost 1 (\% Saving w.r.t. 3/2) & Cost 2 (\% Saving w.r.t. 3/2) & Cost 3 (\% Saving w.r.t. 3/2) \\
\hline $\mathrm{C}_{\mathrm{t}} 1$ & $1050.40(91.82 / 45.38)$ & $1923.18(85.01 / 0)$ & $12833.26(0 /-)$ \\
$\mathrm{C}_{\mathrm{t}} 2$ & $1151.59(91.20 / 43.11)$ & $2024.19(84.53 / 0)$ & $13084.84(0 /-)$ \\
$\mathrm{C}_{\mathrm{t}} 3$ & $1200.44(90.74 / 42.41)$ & $2084.46(83.92 / 0)$ & $12960.70(0 /-)$ \\
$\mathrm{C}_{\mathrm{t}} 4$ & $1177.94(90.84 / 42.61)$ & $2052.35(84.04 / 0)$ & $12862.09(0 /-)$ \\
$\mathrm{C}_{\mathrm{t}} 5$ & $1219.44(90.57 / 41.36)$ & $2079.37(83.92 / 0)$ & $12932.91(0 /-)$ \\
$\mathrm{C}_{\mathrm{t}} 6$ & $1236.12(90.64 / 41.78)$ & $2123.02(83.92 / 0)$ & $13204.72(0 /-)$ \\
$\mathrm{C}_{\mathrm{t}} 7$ & $269.17(97.72 / 71.05)$ & $929.71(92.11 / 0)$ & $11786.42(0 /-)$ \\
$\mathrm{C}_{\mathrm{t}} 8$ & $1750.06(85.80 / 36.01)$ & $2734.69(77.81 / 0)$ & $12323.05(0 /-)$ \\
\hline
\end{tabular}

Table 7: Statistical comparison of algorithms for 31 runs.

\begin{tabular}{|cccccc|}
\hline Cost function $\left(\mathrm{F}_{1}\right)$ & Min & Max & Average & STD & Median \\
\hline PSO & 1042.03 & 1133.50 & 1099.67 & 21.42 & 1102.77 \\
FPA & 1080.17 & 1173.81 & 1115.47 & 24.37 & 1107.14 \\
ALO & 1089.60 & 1195.49 & 1141.13 & 30.10 & 1140.74 \\
GA & 1054.98 & 1141.95 & 1081.04 & 19.40 & 1074.21 \\
NBAT & 1084.19 & 1202.41 & 1149.25 & 29.19 & 1149.70 \\
FA & 1083.38 & 1154.44 & 1113.29 & 17.12 & 1110.83 \\
\hline
\end{tabular}

Finally, several advanced optimisation algorithms were implemented to solve the optimisation problem, but not all the algorithms were able to converge to an effective solution. Thus, only competitive algorithms are demonstrated here for the purpose of a comparison. In our study, the PSO algorithm developed has demonstrated the best performance for solving the optimisation problem among the other options, although the recent algorithms are proven the best among other existing algorithms during their proposals. Generally, the performance of the algorithms depends on the types of problem formulated.

\section{Conclusion}

This paper proposes a day-ahead energy management approach for a community microgrid while considering degradation costs of a battery to minimise its operational costs. In the approach, cycling costs are added to the energy exchange program to demonstrate their effect on the operational costs. The optimisation problem formulated is solved using a developed framework in which the particle swarm optimisation algorithm, whose effectiveness is verified by comparing results with those obtained from other recently developed algorithms, is designed. The impact of uncertainties on output results is analysed. It is demonstrated that real-time electricity price has a direct effect on the scheduling program whereas uncertainty in renewable power generation and power 


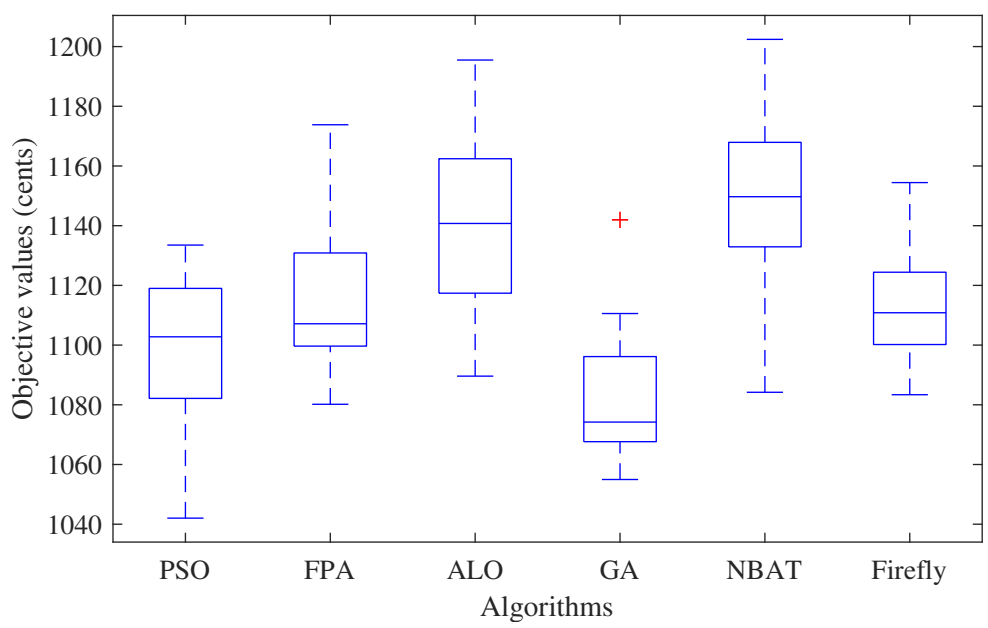

Figure 21: Comparisons of algorithms using box plot for 31 runs.

Table 8: Wilcoxon test for comparing algorithms.

\begin{tabular}{|cccccc|}
\hline Algorithms & PSO vs FPA & PSO vs ALO & PSO vs GA & PSO vs NBAT & PSO vs FA \\
\hline P-value & 0.030151 & $1.3725 \mathrm{e}-06$ & 0.00028094 & $2.1034 \mathrm{e}-08$ & 0.019437 \\
Significant PSO & Yes & Yes & Yes & Yes & Yes \\
\hline
\end{tabular}

demand has no significant effect on the solution. It is shown that including cycling costs to the objective function determines the actual operational costs of the microgrid. It is also revealed that the proposed algorithm can reduce electricity costs around $40 \%$ as compared to the baseline approach. Most importantly, this study also finds that the microgrid can save on average $91.57 \%$ of the operational costs as compared to the only gridconnected distribution network, without renewable energy sources and an energy storage system. Also, it can reduce costs on average by $47.43 \%$ as compared to the distribution network possessing only renewable energy sources.

\section{Acknowledgement}

This work is supported by the Australian Government Research Training Program Scholarship at The University of New South Wales - Canberra, Australia.

\section{References}

[1] G. Cardoso, T. Brouhard, N. DeForest, D. Wang, M. Heleno, L. Kotzur, Battery aging in multi-energy microgrid design using mixed integer linear programming, Applied energy 231 (2018) 1059-1069.

[2] M. A. Hossain, H. R. Pota, W. Issa, M. J. Hossain, Overview of ac microgrid controls with inverterinterfaced generations, Energies 10 (9) (2017) 1300.

[3] M. A. Hossain, H. R. Pota, M. J. Hossain, F. Blaabjerg, Evolution of microgrids with converter-interfaced generations: challenges and opportunities, International Journal of Electrical Power \& Energy Systems 109 (2019) 160-186. 
[4] G. Fridgen, M. Kahlen, W. Ketter, A. Rieger, M. Thimmel, One rate does not fit all: An empirical analysis of electricity tariffs for residential microgrids, Applied energy 210 (2018) 800-814.

[5] C. Ju, P. Wang, L. Goel, Y. Xu, A two-layer energy management system for microgrids with hybrid energy storage considering degradation costs, IEEE Transactions on Smart Grid 9 (6) (2018) 6047-6057.

[6] A. S. Jacob, R. Banerjee, P. C. Ghosh, Sizing of hybrid energy storage system for a pv based microgrid through design space approach, Applied energy 212 (2018) 640-653.

[7] B. Xu, J. Zhao, T. Zheng, E. Litvinov, D. S. Kirschen, Factoring the cycle aging cost of batteries participating in electricity markets, IEEE Transactions on Power Systems 33 (2) (2018) 2248-2259.

[8] C. A. Correa-Florez, A. Gerossier, A. Michiorri, G. Kariniotakis, Stochastic operation of home energy management systems including battery cycling, Applied energy 225 (2018) 1205-1218.

[9] C. Huang, D. Yue, S. Deng, J. Xie, Optimal scheduling of microgrid with multiple distributed resources using interval optimization, Energies 10 (3) (2017) 339.

[10] T. Weitzel, M. Schneider, C. H. Glock, F. Löber, S. Rinderknecht, Operating a storage-augmented hybrid microgrid considering battery aging costs, Journal of Cleaner Production 188 (2018) 638-654.

[11] M. Sedighizadeh, A. H. Mohammadpour, S. M. M. Alavi, A two-stage optimal energy management by using adp and hbb-bc algorithms for microgrids with renewable energy sources and storages, Journal of Energy Storage 21 (2019) 460-480.

[12] J. Li, M. A. Danzer, Optimal charge control strategies for stationary photovoltaic battery systems, Journal of Power Sources 258 (2014) 365-373.

[13] D. Fuselli, F. De Angelis, M. Boaro, S. Squartini, Q. Wei, D. Liu, F. Piazza, Action dependent heuristic dynamic programming for home energy resource scheduling, International Journal of Electrical Power \& Energy Systems 48 (2013) 148-160.

[14] M. Boaro, D. Fuselli, F. De Angelis, D. Liu, Q. Wei, F. Piazza, Adaptive dynamic programming algorithm for renewable energy scheduling and battery management, Cognitive Computation 5 (2) (2013) 264-277.

[15] D. Liu, Y. Xu, Q. Wei, X. Liu, Residential energy scheduling for variable weather solar energy based on adaptive dynamic programming, IEEE/CAA Journal of Automatica Sinica 5 (1) (2018) 36-46.

[16] G. Shi, Q. Wei, D. Liu, Optimization of electricity consumption in office buildings based on adaptive dynamic programming, Soft Computing 21 (21) (2017) 6369-6379.

[17] Q. Wei, F. L. Lewis, G. Shi, R. Song, Error-tolerant iterative adaptive dynamic programming for optimal renewable home energy scheduling and battery management, IEEE Transactions on Industrial Electronics 64 (12) (2017) 9527-9537.

[18] M. A. Hossain, H. R. Pota, S. Squartini, F. Zaman, K. M. Muttaqi, Energy management of community microgrids considering degradation cost of battery, Journal of Energy Storage 22 (2019) 257-269. 
[19] M. A. Hossain, H. R. Pota, S. Squartini, A. F. Abdou, Modified pso algorithm for real-time energy management in grid-connected microgrids, Renewable Energy 136 (2019) 746-757.

[20] A. Kaabeche, M. Belhamel, R. Ibtiouen, Sizing optimization of grid-independent hybrid photovoltaic/wind power generation system, Energy 36 (2) (2011) 1214-1222.

[21] B. S. Borowy, Z. M. Salameh, Optimum photovoltaic array size for a hybrid wind/pv system, IEEE Transactions on energy conversion 9 (3) (1994) 482-488.

[22] K. Steriotis, G. Tsaousoglou, N. Efthymiopoulos, P. Makris, E. M. Varvarigos, A novel behavioral real time pricing scheme for the active energy consumers participation in emerging flexibility markets, Sustainable Energy, Grids and Networks 16 (2018) 14-27.

[23] ComEd, USA, http://www.thewattspot.com/.

[24] H. Hesse, M. Schimpe, D. Kucevic, A. Jossen, Lithium-ion battery storage for the grida review of stationary battery storage system design tailored for applications in modern power grids, Energies 10 (12) (2017) 2107.

[25] X. Luo, J. Wang, M. Dooner, J. Clarke, Overview of current development in electrical energy storage technologies and the application potential in power system operation, Applied energy 137 (2015) 511-536.

[26] M. Petit, E. Prada, V. Sauvant-Moynot, Development of an empirical aging model for li-ion batteries and application to assess the impact of vehicle-to-grid strategies on battery lifetime, Applied energy 172 (2016) 398-407.

[27] A. Marongiu, M. Roscher, D. U. Sauer, Influence of the vehicle-to-grid strategy on the aging behavior of lithium battery electric vehicles, Applied Energy 137 (2015) 899-912.

[28] W. Ying, Z. Zhi, A. Botterud, K. Zhang, D. Qia, Stochastic coordinated operation of wind and battery energy storage system considering battery degradation, Journal of Modern Power Systems and Clean Energy 4 (4) (2016) 581-592.

[29] F. Zaman, S. M. Elsayed, T. Ray, R. A. Sarker, Configuring two-algorithm-based evolutionary approach for solving dynamic economic dispatch problems, Engineering Applications of Artificial Intelligence 53 (2016) $105-125$.

[30] N. Growe-Kuska, H. Heitsch, W. Romisch, Scenario reduction and scenario tree construction for power management problems, in: 2003 IEEE Bologna Power Tech Conference Proceedings,, Vol. 3, IEEE, 2003, pp. $7-$ pp.

[31] J. Kennedy, R. Eberhart, C. 1995. particle swarm optimization, in: IEEE International Conference on Neural Networks (Perth, Australia), IEEE Service Center, Piscataway, NJ, pp. 1942-1948.

[32] V. S. Pappala, I. Erlich, Power system optimization under uncertainties: A pso approach, in: Swarm Intelligence Symposium, 2008. SIS 2008. IEEE, IEEE, 2008, pp. 1-8.

[33] M. AlRashidi, M. AlHajri, A. Al-Othman, K. El-Naggar, Particle swarm optimization and its applications in power systems, in: Computational Intelligence in Power Engineering, Springer, 2010, pp. $295-324$. 
[34] Y. Del Valle, G. K. Venayagamoorthy, S. Mohagheghi, R. G. Harley, J. Hernandez, Particle swarm optimization: basic concepts, variants and applications in power systems, IEEE Transactions on Evolutionary Computation 12 (2) (2008) $171-195$.

[35] N. Kanwar, N. Gupta, K. Niazi, A. Swarnkar, R. Bansal, Simultaneous allocation of distributed energy resource using improved particle swarm optimization, Applied energy 185 (2017) 1684-1693.

[36] M. Clerc, J. Kennedy, The particle swarm-explosion, stability, and convergence in a multidimensional complex space, IEEE transactions on Evolutionary Computation 6 (1) (2002) 58-73.

[37] C. Amzallag, J. Gerey, J. Robert, J. Bahuaud, Standardization of the rainflow counting method for fatigue analysis, International journal of fatigue 16 (4) (1994) 287-293.

[38] F. Zaman, R. Sarker, G. Chang, Dynamic scenario-based solution approach for scheduling solar-thermal generators, in: 2017 2nd IEEE International Conference on Computational Intelligence and Applications (ICCIA), IEEE, 2017, pp. 492-497.

[39] X.-S. Yang, Flower pollination algorithm for global optimization, in: International conference on unconventional computing and natural computation, Springer, 2012, pp. 240-249.

[40] S. Mirjalili, The ant lion optimizer, Advances in Engineering Software 83 (2015) 80-98.

[41] J. McCall, Genetic algorithms for modelling and optimisation, Journal of Computational and Applied Mathematics 184 (1) (2005) 205-222.

[42] X.-B. Meng, X. Z. Gao, Y. Liu, H. Zhang, A novel bat algorithm with habitat selection and doppler effect in echoes for optimization, Expert Systems with Applications 42 (17-18) (2015) 6350-6364.

[43] X.-S. Yang, Firefly algorithms for multimodal optimization, in: International symposium on stochastic algorithms, Springer, 2009, pp. 169-178. 